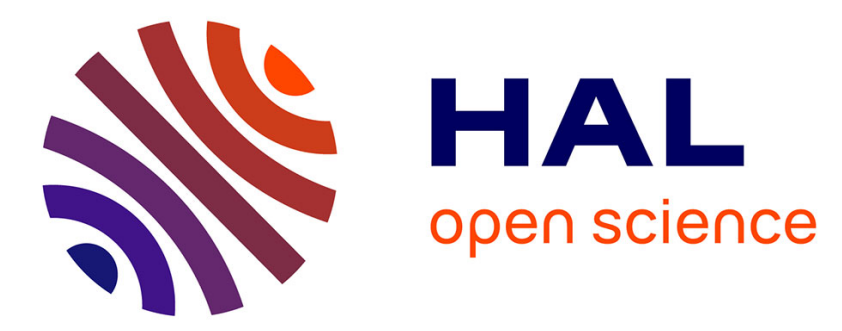

\title{
Potential Time Domain Model with Viscous Correction and CFD Analysis of a Generic Surging Floating Wave Energy Converter
}

\author{
Majid Bhinder, A. Babarit, L. Gentaz, Pierre Ferrant
}

\section{- To cite this version:}

Majid Bhinder, A. Babarit, L. Gentaz, Pierre Ferrant. Potential Time Domain Model with Viscous Correction and CFD Analysis of a Generic Surging Floating Wave Energy Converter. International Journal of Marine Energy, 2015, 10, pp.70-96. 10.1016/j.ijome.2015.01.005 . hal-01145198

\author{
HAL Id: hal-01145198 \\ https://hal.science/hal-01145198
}

Submitted on 8 Jun 2019

HAL is a multi-disciplinary open access archive for the deposit and dissemination of scientific research documents, whether they are published or not. The documents may come from teaching and research institutions in France or abroad, or from public or private research centers.
L'archive ouverte pluridisciplinaire HAL, est destinée au dépôt et à la diffusion de documents scientifiques de niveau recherche, publiés ou non, émanant des établissements d'enseignement et de recherche français ou étrangers, des laboratoires publics ou privés. 


\title{
Potential time domain model with viscous correction and CFD analysis of a generic surging floating wave energy converter
}

\author{
Majid A. Bhinder*, Aurélien Babarit, Lionel Gentaz, Pierre Ferrant
}

Laboratoire d'Hydrodynamique, Energétique et Environement Atmosphérique (LHEEA), UMR CNRS 6598, LUNAM Université,

Ecole Centrale de Nantes, Nantes, France

The state of the art tools to assess the efficiency of the wave energy converters comprise the boundary element method (BEM) codes which are based on the potential linear approach whereas computational fluid dynamics (CFD) is still considered to be relatively computationally expensive. An attempt to enlarge the scope of the state of the art computational tools for wave energy converter applications is made in order to account for the viscous effects. This is achieved via the viscous damping term of the Morison equation which relies on a coefficient $C_{d}$ - to be estimated prior force calculation.

The state of the art wave to wire model together with additional viscous term is termed as potential time domain viscous model and is employed for evaluation of the power efficiency of a generic surging type wave energy conversion system. Finally a comparison of CFD and the viscous time domain model is conducted which concludes that the Morison equations' drag term does offer an improvement.

\section{Introduction}

The research in wave energy devices; with first patent that dates back to 1799 (Girard and his son, France) [1]; has a considerable research and development record spanning past few decades. Over the

\footnotetext{
* Corresponding author at: Beaufort, HMRC, University College Cork, Ireland.

E-mail addresses: mbhinder@ucc.ie (M.A. Bhinder), Aurelien.Babarit@ec-nantes.fr (A. Babarit), Lionel.Gentaz@ec-nantes.fr (L. Gentaz), Pierre.Ferrant@ec-nantes.fr (P. Ferrant).
} 
recently past few years wave energy sector has undergone a remarkable rise in funding sources and consequently a significant development in this field is observed. A number of projects are underway and wind plus wave based combined platforms are currently being proposed in order to combat the energy issue with renewable solution. Some worth mentioning review studies regarding wave energy and wave energy technologies include: [2-6]. Present work is an attempt towards improvement of the computational numerical analysis aimed at assessing power absorption capability of floating point absorber type wave energy converter.

The focus of present study has been devoted to the viscous drag forces that exist as the floating structures interacts with surrounding fluid in particular fluctuating wave forces. A number of researchers; [7-9]; have mentioned that in the numerical analysis the viscous forces has been taken into account according to the viscous drag damping. However the quantification of the power loss in the absence of this additional viscous term is usually omitted while the drag coefficient is usually taken from the existing experimental data.

Incorporation of a dissipation force in a BEM solver is reported in [10] and it is demonstrated that such technique is useful in achieving additional damping for the floating body in particular in the resonance zone while the coefficient of this dissipation term rely on the experiments or CFD computations. The existing data, mostly experimental, carry a huge scatter in the value of the force coefficients and usually a difference of $30-40 \%$ is seen in the reported values [11, chapter 12]. Therefore the prediction of the drag coefficient for a complex geometrical structure poses a major issue. In [12,13] it is explored and is demonstrated that the CFD can be employed for such objective. This study investigates the methodology of using the Morison equation into the wave to wire numerical model of wave energy converters.

Fig. 1 shows a schematic of the rectangular generic surging wave energy converter (WEC). A full scale model with dimensions; height $(h)=20 \mathrm{~m}$ and draft $(z$-axis $)=10 \mathrm{~m}$ and width $(\mathrm{w})=7.85 \mathrm{~m}$ and length $(\mathrm{l})=10 \mathrm{~m}$ is considered for this study.

A wave to wire model of a single degree of freedom surging buoy has been derived from the equation of motion. This model is termed here as potential time domain viscous (PTDV) model. To help understand the importance of the viscous forces regarding considered wave energy converters, the outcome of the PTDV model is compared against the findings of the CFD solver. A such analysis shows how a simple wave structure interaction model using potential theory can be improved when viscous drag term is included into the wave to wire model consequently validating the PTDV model. To accomplish this task, CFD computations for the following three flow scenarios are conducted;

1. Radiation: rigid structure (wave energy converter) is made to oscillate in fluid at rest.

2. Diffraction: waves are interacting with the wave energy converter held still in a numerical wave tank. The rigid structure then behaves as an obstacle to the wave field and causes a wave diffraction field.

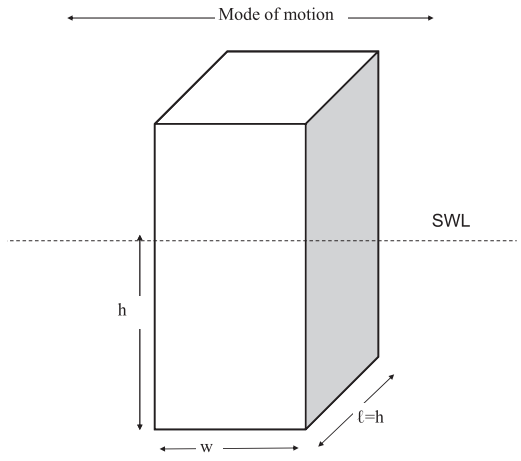

Fig. 1. Schematic of generic WEC. 
3. Wave structure interaction (WSI): waves are generated and the wave energy converter is free to respond to the incoming wave forces.

\subsection{Viscous forces}

The wave energy converters (especially the floating type - point absorbers) are designed to produce electricity as they interact with the surrounding wave climate. Therefore it becomes greatly important that, for physical and numerical testing, the wave forces are accurately measured and/or calculated. The problem of wave structure interaction, in its simplest form, is usually studied in terms of the oscillatory flow past a rigid structure.

The experimental set-up of oscillating flow problems usually consists of large U-tube apparatus where fluid is made to oscillate at a certain required frequency while the rigid structure is held fixed. A second method of studying such a problem is to impose oscillatory motion on the rigid structure in an otherwise calm fluid. The inertia coefficient of former and lateral approach is usually denoted by $C_{\mathrm{m}}$ and $C_{\mathrm{I}}$, respectively, with $C_{\mathrm{m}}=1+C_{\mathrm{l}}$, this is because the inertia force(s) in the two methods are not the same. ${ }^{1}$

The viscous drag force evaluation for simple shaped structures like cylinder and square with smooth and sharp corners have been the subject of a number of studies from the last few decades. For example the analysis of the viscous oscillatory flow can be traced back to [14]. Sarpkaya [15] has discussed the viscous effects in relation to low Keulegan-Carpenter (KC) numbers. It has been emphasized in a number of studies (for example [16]) that not only is the KC number important to the oscillatory flow analysis but the viscous parameter named $\beta$ should also be studied alongside KC number. This viscous parameter $\beta$ is defined as the ratio of the Reynolds number to the Keulegan-Carpenter number. A very low value of the $\beta$ represents a very small scale model laboratory experiment/numerical test. The KC number is defined as

$$
\mathrm{KC}=\frac{U_{\mathrm{m}} T}{D}
$$

here $U_{\mathrm{m}}=$ amplitude of the sinusoidal velocity, $T$ = time period of the sinusoidal velocity, and $D=$ relevant dimension of the rigid structure. For deep water waves the KC number can be re-written as

$$
\mathrm{KC}=\frac{2 \pi A_{\mathrm{w}}}{D},
$$

where $A_{\mathrm{w}}$ is equal to the amplitude of the wave.

Since the magnitude of the viscous force is directly relevant to the KC number therefore it is worth exploring what range of $\mathrm{KC}$ numbers are relevant to the wave energy converter in question. From the wave statistics data, the maximum significant wave height corresponding to five wave site locations across Europe is shown in Table 1, where $D$ is the relevant dimension of the WEC and taken as equal to the submerged draft of devices i.e., $10 \mathrm{~m}$. Following Eq. (2), a higher wave amplitude would correspond to a higher value of KC. Thus the upper limit of the KC number that corresponds to the wave energy devices is less than 5 (i.e., $\mathrm{KC} \leqslant 5$ ).

\subsection{Morison equation}

Morison [17] proposed a semi-empirical expression that offers the calculation of the in-line force exerted by the oscillatory flow on a slender cylinder. Morison equation provides a semi-empirical formulation to model the unsteady force on rigid structures in oscillatory flow. According to this simple expression the total in-line force on an immersed object within an oscillatory viscous fluid with velocity $\dot{X}$ and acceleration $\ddot{X}$ is expressed as a summation of two components:

- force due to the inertia; an effect of the irrotational (potential) assumption, i.e., $\rho V C_{1} \ddot{X}$.

\footnotetext{
${ }^{1}$ In still water the absence of ambient dynamic pressure gradient leads to a zero Froude-Krylov force.
} 


\section{Table 1}

Maximum significant wave height and corresponding KC value for various locations.

\begin{tabular}{lcll}
\hline Location & $H_{\mathrm{s}-\max }[\mathrm{m}]$ & $D[\mathrm{~m}]$ & $\mathrm{KC}$ \\
\hline Yeu & 7 & 10 & 2.2 \\
SEM-REV & 9 & 10 & 2.8 \\
EMEC & 11 & 10 & 3.4 \\
Lisboa & 7 & 10 & 2.2 \\
Belmullet & 14 & 10 & 4.4 \\
\hline
\end{tabular}

- force due to the viscous drag; effect of the skin friction and flow separation. i.e., $\frac{1}{2} \rho A C_{d} \dot{X}|\dot{X}|$.

For a 3 dimensional structure moving in-line to the oscillatory flow of velocity $U$ and acceleration $\dot{U}$, the Morison equation becomes [18]:

$$
F(t)=-\frac{1}{2} \rho A C_{\mathrm{d}}(\dot{X}-U)|\dot{X}-U|-\rho V C_{\mathrm{I}}(\ddot{X}-\dot{U})+\rho V \dot{U} .
$$

The first term on the right hand side of Eq. (3), is the drag force, second term is hydrodynamic mass force and third term is the Froude-Krylov force. Here $C_{\mathrm{d}}$ is the drag coefficient and $C_{\mathrm{I}}$ the inertia coefficient. $A$ is the relevant cross-sectional area and $V$ the volume of the structure. The inertial force proposed by the Morison equation is proportional to the acceleration of the flow whereas the viscous drag part is proportional to the time dependent flow velocity and acts in the direction of the velocity. The derivation of these coefficients have a record of extensive research both theoretical and experimental. However growing computational capability and development in computational fluid dynamics has lead to the numerical prediction of these coefficient and the existence of experimental data has served as validation tool for advances made in numerical modeling techniques. The somewhat simple look of the Morison equation invited researchers to further improve its scope. This was done by proposing some extra non-linear terms. Keulegan and Carpenter [19] were the first to attempt a correction to the Morison equation.

Cook [20] has reported that despite being imperfect and restricted to further limitations, there appears to be a consensus that the Morison equation is a "good point of departure" when modeling hydrodynamic loadings in oscillatory flow. Furthermore this statement holds true to date. Other early work towards the improvement of the Morison equation regarding vorticity effects include [15,21]. Lighthill [22] proposed correction terms associated with irrotational flow; this attempt is usually remembered as the Lighthill correction. Cook [20] has analyzed the Lighthill correction through experimental data and concludes that the additional terms proposed by the Lighthill correction do not make a significant difference when the Morison force is made to fit the experimental measurements and can therefore be neglected.

When employing the Morison equation for hydrodynamic loading, it is always advantageous to recall previous work done in relation to the limitations of this empirical equation. Some limitations of the Morison equation as stated in [20] and in [18] are listed below:

1. The equation produces approximate profile of the in-line force only.

2. Ratio of the cylinder diameter to flow wave length should be $<2$. This insures that the effect of cylinder motion on incoming waves is ignorable.

3. The equation works favorably within inertia dominated and the drag dominated regions, i.e., $\mathrm{KC}<6$ and $\mathrm{KC}>20$, respectively.

4. The effect of the transverse force is not included.

5. Vortex and wake-return-impact are beyond the scope of the Morison equation.

The flow properties mentioned in the last two limitations (numbered 4 and 5) are usually responsible for the vibrational spikes that are usually observed in experimental results. This fact has been pointed-out by Sumer and Fredsøe [18]. Despite the above mentioned limitations and the approximate 
representation of fluid forces, the Morison equation has enjoyed widespread use. Consequently, there exist a great deal of work done regarding the evaluation of the appropriate values of the force coefficients.

The next section reviews the literary work regarding force coefficient for a cylindrical and a rectangular square structure.

\subsection{Review of work concerning Morison equation coefficients}

The evaluation of the Morison force coefficients usually involves physical laboratory tests to measure the in-line force(s). However some researchers have shown that computational numerical analysis can be adopted as an alternative to the experimental procedure and the force coefficients be determined thus.

For a given flow problem, once the force time history is measured through experiments, we have two forces: one is the experimental and the other the Morison force. The task therefore is to predict those values of the drag and inertia coefficients such that the lateral force (the Morison) is as close to the former force curve as possible. Should a CFD analysis be conducted instead of an experimental procedure, the Morison force curve would need to correspond with the CFD results (for example see [12,13]).

In order to yield an appropriate value of the drag and inertia coefficients there exist a number of methods that can be used for this curve fitting procedure such as Morison's method, Fourier series approach, least squares method, and weighted least squares method.

A brief introduction to these methods can be found in [11]. Some of these methods are considered relatively more accurate. In this study the time invariant value of the drag and inertia coefficient has been evaluated using least squares method.

One might reason that there exist other methods such as the weighted least squares method which offers improved results although more computationally demanding. But the objective of present work is to locate an estimate of the drag coefficient and to study, using a potential time domain viscous model, the variation of the annual power production of the device verses a range of the drag coefficients. For this the drag coefficient value is successively increased starting from its initial nominal value. A comparison of the evaluated drag coefficient with existing literature (see $[12,13]$ ) showed a good match which favors the choice of using the least squares method. A usual least square technique produces the force coefficients by minimizing the error function $E_{\mathrm{r}}$ which is given by:

$$
E_{\mathrm{r}}=\sqrt{\sum_{1}^{N}\left(F_{C F D}-F_{\text {Morison }}\right)^{2}}
$$

Here $N$ is the total number of data sets, and $F_{\text {subscript }}$ is the force corresponding to the subscript. This approach can either be applied on a wave by wave basis or for the whole set of results and produces time invariant force coefficients. In the modified least square method, called weighted least square, the effect of small forces on resulting force coefficients is reduced hence, the method claims to be more accurate in some cases [23].

In [24] theoretical and experimental results regarding viscous oscillatory in-line force on circular and square cylinders are presented. A flow regime of a low KC number and moderately higher $\beta$ parameter is considered in this study. It has been mentioned that when the fluid is oscillating while the cylinder is at rest, the inertia force is increased by the Froude-Krylov force caused by the pressure gradient and this is the reason why the inertia coefficient of this scenario is usually written as $C_{\mathrm{m}}=C_{i}+1$. Following [24] the average values of the drag and inertia coefficient for a circular cylinder can be written as:

$1 \leqslant C_{\mathrm{d}} \leqslant 2$ for $1 \leqslant \mathrm{KC} \leqslant 10$ at three $\beta$ values of 1665,482 and 196 .

$2 \leqslant C_{\mathrm{m}} \leqslant 2.2$ for $1 \leqslant \mathrm{KC} \leqslant 4$ at three $\beta$ values of 1665,1204 and 964 .

For sharp edged plate, square and diamond shaped structures the drag coefficient is reported to be $6 \leqslant C_{\mathrm{d}} \leqslant 7,4 \leqslant C_{\mathrm{d}} \leqslant 5$ and $2 \leqslant C_{\mathrm{d}} \leqslant 3$, respectively, whereas the KC range corresponding to 
these values was found to be $1 \leqslant \mathrm{KC} \leqslant 10$ with a maximum $\beta$ of about 430 . It has been reported that for sharp edged bodies the vortex shedding occurs at even low KC regimes.

In [23], physical model test of a vertical circular cylinder are presented in the context of the Morison force coefficients. A small scale model of the vertical cylinder was tested for the in-line force(s) as waves pass overhead. Moreover two least square methods are discussed in relation to their corresponding impact on the resulting value of the force coefficients.

In [25] numerical computations are made in order to deduce the Morison force coefficients. The test case comprised a square cylinder. The numerical case studies mentioned include two different attach angles of the approaching flow and it has been reported that in case of the zero attack angle the Morison force could not accurately predict the lift force. However the calculations for the in-line drag force were adequately predicted. The numerical methodology implemented was based on a weakly compressible flow method with three-dimensional large eddy simulations.

In [26] numerical results for oscillatory flow past a cylinder with round and with sharp corners are presented and an average value for the drag coefficient is reported to be around 2.9. Numerical computations are shown along experimental results of [27].

Atluri et al. [28] reported a CFD examination of the viscous in-line force for thin sharp edged square plates - the heave plates. CFD results have been presented along side existing experimental data and the advantages of using CFD, such as the ability of readily jump to various scale models and quick assessment of the design variables, are highlighted.

Following the work discussed above it is quite clear that the empirical coefficient required for the drag term of the Morison equation for a circular cylinder resides in between a minimum value of about 1 and a maximum upper value of about 4 whereas for a sharp edged square cylinder or flat plat the lower value of the drag is reported to be about 2 and higher value of about 10 . The $\mathrm{KC}$ range considered lies somewhere less than 6 . It is noted that the higher drag value corresponds to a KC value of less than 1 whereas the lower drag value corresponds to a higher KC number.

\subsection{Potential time domain viscous (PTDV) model}

A wave to wire model of a single degree of freedom surging buoy has been derived from the equation of motion. Equations for the computation of annual power of this one degree of freedom buoy are shown below. From frequency domain analysis the power function of the WEC is obtained from Eq. (5)

$$
P(\omega)=\frac{1}{2} B_{\mathrm{pto}} \omega^{2} \mathrm{RAO}^{2} .
$$

Here RAO is the response amplitude operator of the device. In frequency domain it can be defined as;

$$
\mathrm{RAO}=\left[-\omega^{2}(m+A)+i \omega\left(B+B_{\mathrm{pto}}\right)+K_{11}\right]^{-1} F_{\mathrm{e}} .
$$

Here $A$ is the added mass coefficient, $B$ is the radiation damping coefficient whereas $B_{\text {pto }}$ comes from the power take off (PTO) force and $K_{11}$ being the total stiffness of the system. This total stiffness may include the additional stiffness of the PTO system, hydrostatics contributions and any other additional external force like moorings etc. Likewise additional damping part can be summed into the second term on the right-hand-side of the Eq. (6). It is worth mentioning at this stage that the PTO force that is considered in this study comprises only on the damping part. For derivation of the above relation for the RAO, consider the standard equation of motion i.e.,

$$
F=m \ddot{x}
$$

Where $m$ the mass and $x$ is the displacement caused by the force $F$. In frequency domain analysis the total net force $F$ for a floating structure (with frequency dependent displacement $x$, velocity $\dot{x}$ and acceleration $\ddot{x}$ ) can be written as sum of the three major forces named the radiation force $F_{\mathrm{r}}$, hydrostatic force $F_{\mathrm{h}}$ and the power take off force $F_{\mathrm{pto}}$.

$$
F=F_{\mathrm{r}}+F_{\mathrm{h}}+F_{\text {pto }}
$$




$$
\begin{aligned}
& F_{\mathrm{r}}=-A \ddot{x}-B \dot{x} \\
& F_{\mathrm{h}}=-k_{\mathrm{h}} x \\
& F_{\mathrm{pto}}=-K_{\mathrm{pto}} x-B_{\mathrm{pto}} \dot{x} .
\end{aligned}
$$

Here $k_{\mathrm{h}}$ is hydrostatic stiffness and $K_{\text {pto }}$ is the stiffness contribution from the PTO. Substituting Eqs. (8)-(11) into Eq. (7) yields Eq. (6), with $K_{11}=K_{\mathrm{pto}}+k_{\mathrm{h}}$. From the power function (Eq. (5)) the mean power of a sea state for given spectrum $S\left(f_{i}\right)$ is obtained from (Eq. (12)) to be

$$
\overline{P_{f}}\left(H_{s}, T_{p}\right)=\sum_{i=1}^{N} 2 P\left(f_{i}\right) S\left(f_{i}\right) \Delta f .
$$

with $\Delta f$ being the frequency step used in the discretization of the spectrum $S$. And $i$ being the index of the frequency (or angular frequency) of the waves.

Then the annual power production (APP) of the wave energy conversion system at a given location is obtained through the product of the sea statistics data obtained from the scatter diagram (say $C\left(H_{s}, T_{p}\right)$ ) of the deployment site and the above mentioned mean power of the considered spectrum, as shown in Eq. (13),

$$
P_{\mathrm{APP}}(\omega)=\sum_{k=1}^{N T} \sum_{j=1}^{N H} \overline{P_{f}}\left(H_{s, j}, T_{p, k}\right) C\left(H_{s, j}, T_{p, k}\right),
$$

where $N T$ and $N H$ are the indices of the maximum values of $T_{p}$ and $H_{s}$, respectively. For the Yeu island site (located near coast of western France) the significant wave height and the peak wave period considered here comprises:

$$
\begin{aligned}
& 0.5 \leqslant H_{s} \leqslant 7.5 \\
& 3.0 \leqslant T_{p} \leqslant 17 .
\end{aligned}
$$

The APP of the device for non-linear analysis should be computed via time domain analysis. In time domain the equation of motion of the buoy in single degree of freedom (say surge) is (using the Cummins relation [29]).

$$
\left(M+\mu_{\infty}\right) \ddot{X}(t)=F_{\text {ex }}(t)-\sum_{i=1}^{N_{\text {prony }}} I_{i}-B_{\text {pto }} \dot{X}(t)-K_{11} X(t)+\text { viscous drag-term. }
$$

Here $M$ the mass of device, $\mu_{\infty}$ is the added mass obtained from frequency domain coefficients, and $F_{\mathrm{ex}}(t)$ is the wave excitation force in time domain. $K_{11}$ is the total stiffness and viscous drag-term is the drag component of the Morison equation, i.e., $\frac{1}{2} \rho A C_{\mathrm{d}} U_{\mathrm{r}}\left|U_{\mathrm{r}}\right|$. It is worth noticing that $U_{\mathrm{r}}$ is the relative velocity of the float with respect to the velocity of the incoming waves, that is $U_{\mathrm{r}}=\dot{X}-V$, where $\dot{X}$ is the instantaneous velocity of the buoy as it moves in response to the waves and $V$ is the corresponding incoming wave velocity taken in accordance to the linear wave theory for deep water scenario. For the surging case this horizontal velocity component of the wave velocity is given by Eq. (17). In Eq. (16) the convolution product of radiation impulse response function times the velocity has been replaced by an additional state variables $I_{i}$ using the methodology described in [30], these state variables are shown in Eq. (18) where $\alpha$ and $\beta$ are the amplitude and the phase of the complex coefficients, respectively and $N_{\text {prony }}$ is the total number of these coefficients.

$$
V=\omega A_{\text {wave }} e^{k z} \sin (\omega t-k x)
$$

here $A_{\text {wave }}$ is wave amplitude, $t$ the time vector and $k$ being the wave number.

$$
\begin{gathered}
\dot{I}_{1}=\beta_{1} I_{1}+\alpha_{1} \dot{X} \\
\vdots \\
\dot{I}_{N_{\text {prony }}}=\beta_{N_{\text {prony }}} I_{N_{\text {prony }}}+\alpha_{N_{\text {prony }}} \dot{X}
\end{gathered}
$$


In the time domain the waves corresponding to the sea state $\left(H_{s}, T_{p}\right)$ are described as a superposition of regular waves and the resulting elevation of the irregular wave is (Eq. (19))

$$
\eta(t)=\sum_{i=1}^{N \text { wave }} A_{\text {wave }, i} \cos \left(\omega_{i} t+\varphi_{i}\right)
$$

here $\varphi_{i}$ are random phases between $[0-2 \pi], i$ is the index of the wave frequency, and Nwave being the total number of summed waves.

$$
A_{\text {wave }, i}=\sqrt{2 S\left(H_{s}, T_{p}, f_{i}\right) \Delta f} .
$$

The excitation force due to irregular wave of the defined spectrum is then given by

$$
\left.F_{\text {ex }}(t)=\mathfrak{R} \sum_{i=1}^{N \text { wave }} A_{\text {wave }, i} F_{\text {ex }}\left(\omega_{i}\right) e^{i\left(\omega_{i} t+\varphi_{i}\right)}\right) .
$$

Once the time domain equation of motion is solved for $\dot{X}$, the instantaneous power $P_{\text {ins }}(t)$ is

$$
P_{\text {inst }}(t)=B_{\text {pto }} \dot{X}(t)^{2}
$$

and the mean power:

$$
\overline{P_{T}}\left(H_{s}, T_{p}\right)=\frac{1}{T} \int_{0}^{T} P_{\text {inst }}(t) \mathrm{d} t,
$$

where $T$ is the total duration of the simulation. Similar to the frequency domain now the APP of this time domain model is obtained by multiplying the mean power $\overline{P_{T}}$ by the sea statistics say $C\left(H_{s}, T_{p}\right)$ i.e.,

$$
P_{\mathrm{APP}}(t)=\sum_{k=1}^{N T} \sum_{j=1}^{N H} \overline{P_{T}}\left(H_{s, j}, T_{p, k}\right) C\left(H_{s, j}, T_{p, k}\right)
$$

\subsubsection{Computation setup and results}

For the computations of the linear hydrodynamic coefficients three mesh configurations are initially simulated. Results of these three mesh profiles are compared and the corresponding plots are presented in Fig. 2. Only the submerged part of the float is meshed for computations of the hydrodynamic parameters using Aquaplus. Aquaplus is BEM solver for calculation of first order hydrodynamic coefficients which has been developed at the Ecole Centrale de Nantes, France [31]. One can see that a perfect agreement, of response amplitude operator (RAO) of the device for each mesh, confirms that the results are mesh independent. Following this a mesh with 500 panels was chosen for the rest of the computations as presented from here onwards. Table 2 presents the set of the input parameters that were used in the Aquaplus computations and the frequency dependent added mass A, radiation damping B and excitation force Fe for the surge motion of the float are shown in Fig. 3.

The overall picture of the performance of the WEC is shown via a numerically computed power matrix as can be seen in Fig. 4, which shows the average value of power production for each corresponding set of $H_{s}$ and $T_{p}$. It is reasonably prominent that when viscous damping is taken into account the predicted performance is reduced to almost $64 \%$ of the initial power production which was computed without the additional viscous drag force. The corresponding absorbed power as a function of the wave frequency is shown in Fig. 5 where the lower peak of the absorbed power refers to a viscous force scenario.

The numerical tests for the APP of the WEC regarding three test locations were performed. The resulted APP for various values of the drag coefficient at three test sites are shown in Fig. 6. A significant impact of the drag force in relation to the APP is evident from these results.

Further a possible solution to improve power efficiency, in such a case where drag is causing a steep reduction of the power output, has been proposed. This concerns the tuning of the power take 


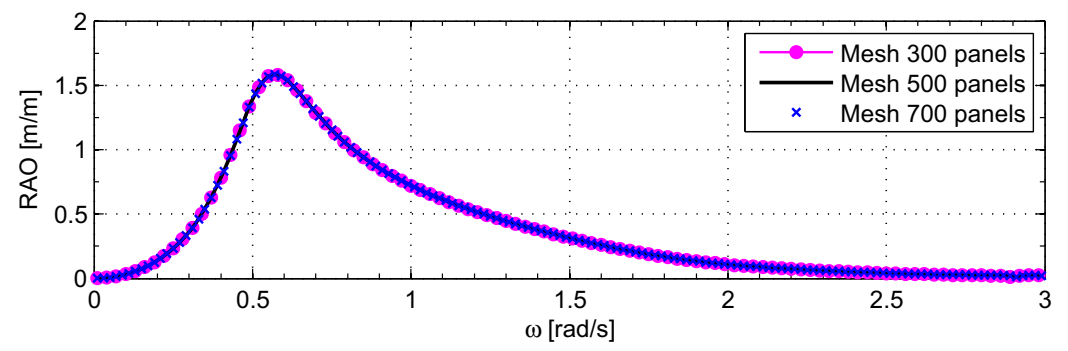

Fig. 2. Mesh convergence test for Aquaplus.

Table 2

Input parameters for surging WEC.

\begin{tabular}{lll}
\hline Parameter & Units & Value \\
\hline$B_{\text {pto }}$ & {$[\mathrm{N} . \mathrm{s} / \mathrm{m}]$} & 500,000 \\
$M$ & {$[\mathrm{~kg}]$} & 785,000 \\
$K_{11}$ & {$[\mathrm{~N} / \mathrm{m}]$} & 500,000 \\
\hline
\end{tabular}
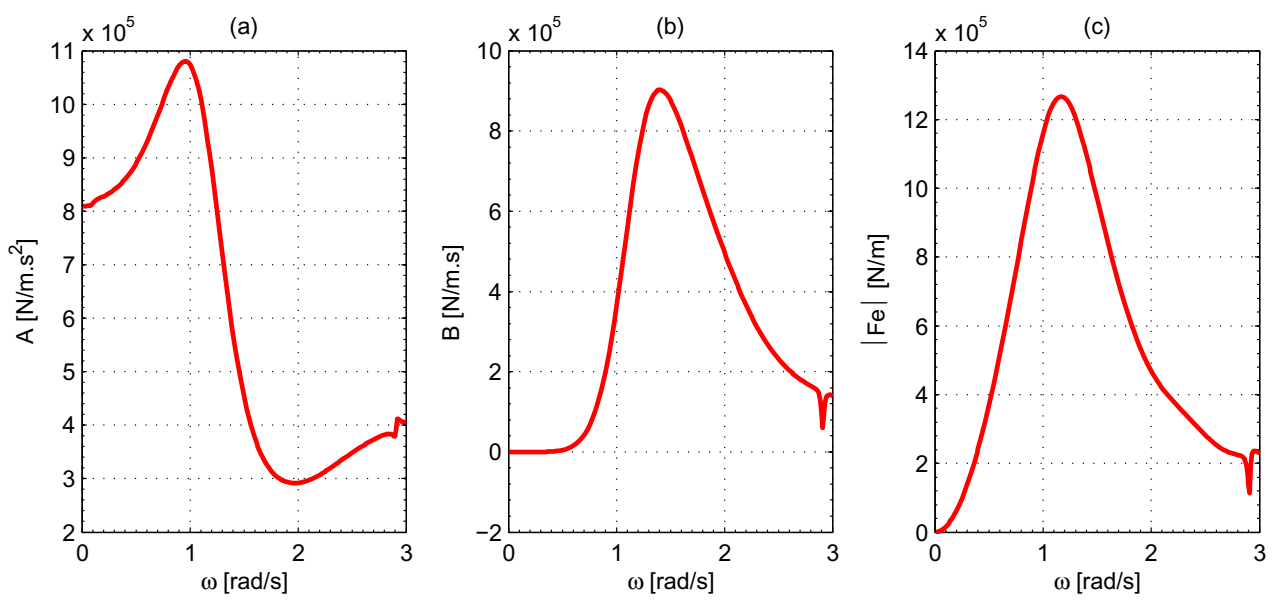

Fig. 3. Hydrodynamic parameters for surging WEC (a) added mass, (b) radiation damping and (c) excitation force.

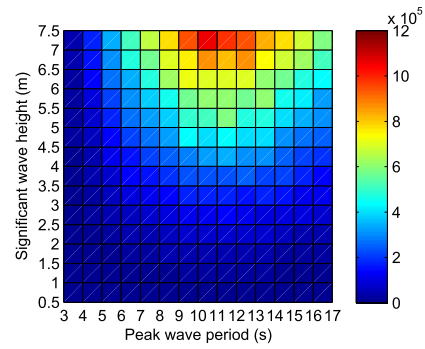

(a) without $C_{d}$

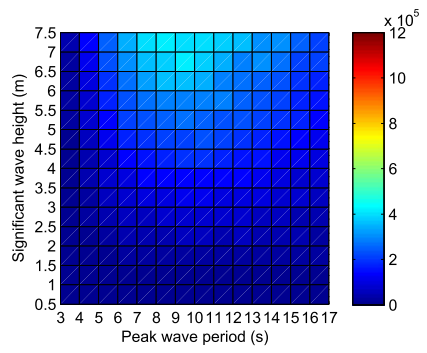

(b) with $C_{d}$

Fig. 4. Power matrix without $C_{\mathrm{d}}$ and with $C_{\mathrm{d}}(=1.8)$, showing power in [Watts]. 


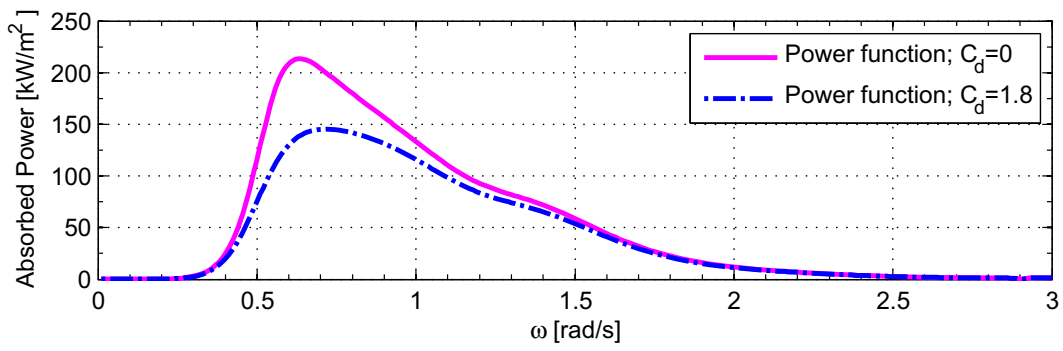

Fig. 5. Power function with drag coefficient and without drag coefficient.

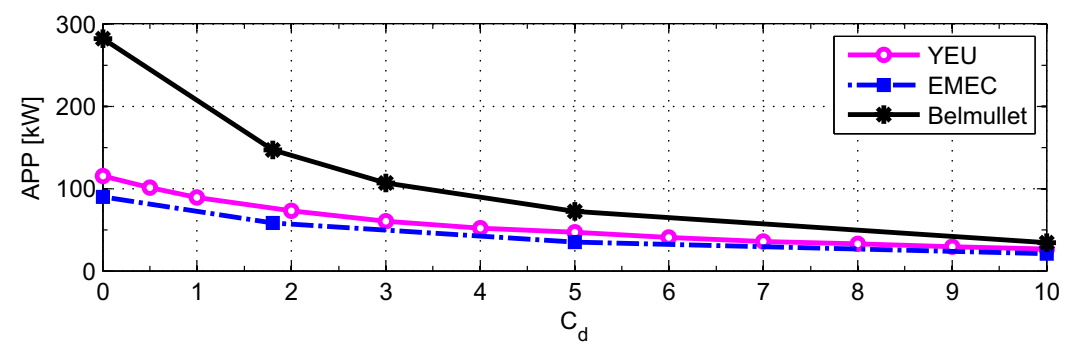

Fig. 6. APP at three test locations as a function of the $C_{\mathrm{d}}$.

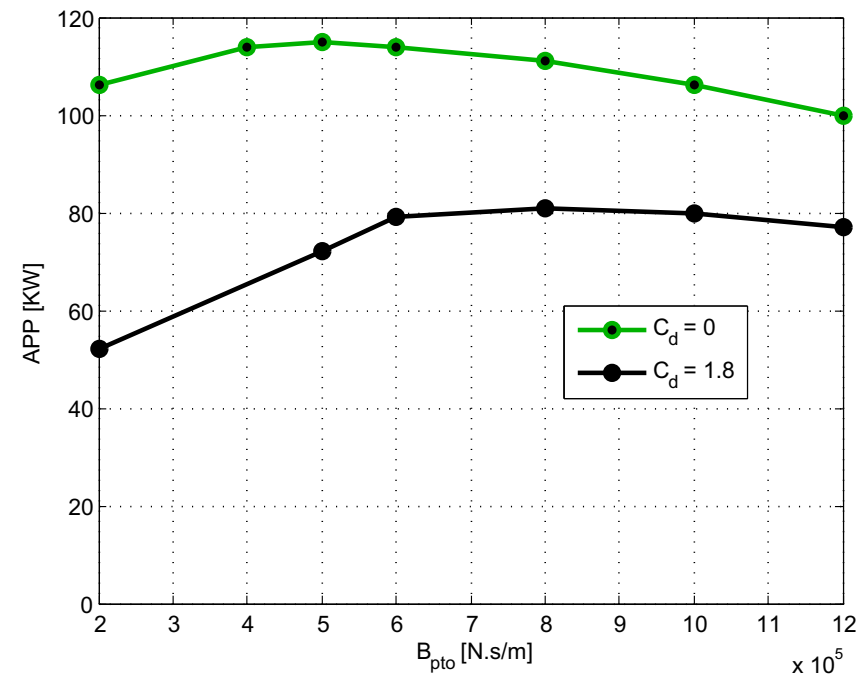

Fig. 7. Variation of APP against PTO damping for surging WEC.

off system. Fig. 7 demonstrates that a variation of the employed PTO damping can improve the power output even when the drag force has been included in the computational model.

\subsection{CFD - FLOW3D}

FLOW3D is a RANSE (Reynolds Averaged Navier-Stokes Equations) solver. The fluid in FLOW3D is defined using a volume of fluid (VOF) function $\mathcal{F}$ [32]. For single fluid, $\mathcal{F}$ is the volume fraction 
occupied by the fluid and thus is equal to 1 and for the void region (the region above free surface in this case) the value of $\mathcal{F}$ is 0 . Here void region is area of the computational domain where a uniform pressure (atmospheric pressure) is assigned.

\subsubsection{Simulation setup}

Table 3 shows the main solver properties for the three dimensional simulations conducted in FLOW3D. A detailed summary of the mesh used, boundary conditions, turbulence models and the convergence test of FLOW3D is presented next.

1.5.1.1. Meshing. In FLOW3D the technique that defines the geometry of the solid structures is named as FAVOR ${ }^{\mathrm{TM}}$. This technique is based on the concept of area fraction (AF) and volume fraction (VF) of the rectangular structured mesh. Thus the geometry shape depends on the mesh cells that lie on the boundary of the structure. As the shape of the rigid body depends on the area and volume fractions of occupied cells, a local fine mesh was needed to establish the exact geometric shape of the rigid body. An optimum mesh was obtained by adding extra fixed points in the vicinity of the WEC. The way how this mesh renders the geometrical shape of the rigid body is further explained with the help of an illustration; see Fig. 8, where the geometrical shape corresponding to three different mesh cell sizes is shown and it can been seen that to obtain a desired geometry shape, with higher precision, a much refined cell size would be required. In the present simulations, the sharp edges of the wave energy converter are to play a very important role in generating vortices, which in turn would influence the total viscous drag force(s). Therefore a fixed point method of the FLOW3D mesh generation was used and a smaller cell size at the fixed point insured that flow field in this region of oscillating motion would be resolved, this insured that the shape of the body edges will not be lost as the structure oscillates.

Table 3

General model properties.

\begin{tabular}{ll}
\hline Flow mode & Incompressible \\
Number of fluid & One fluid \\
Moving object model & Implicit \\
Turbulent model & Renormalized group (RNG) model \\
Pressure solver & Implicit, GMRES \\
Volume of fluid advection & Split Lagrangian method \\
Momentum advection & Second order monotonicity preserving \\
\hline
\end{tabular}

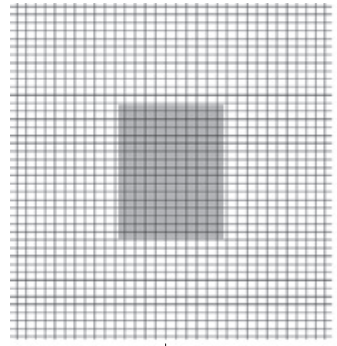

(a)

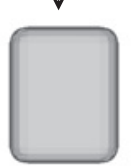

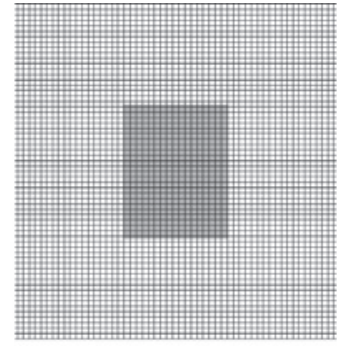

(b)

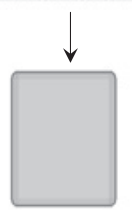

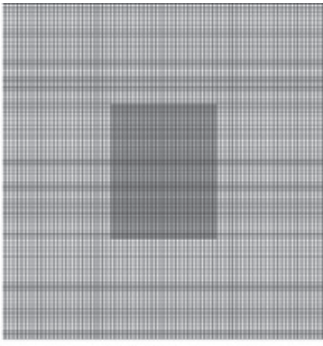

(c)

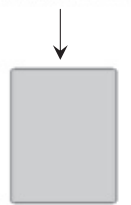

Fig. 8. Three mesh structures and corresponding rendered geometry; (a) cell size $0.6 \mathrm{~m}$, (b) cell size $0.4 \mathrm{~m}$, (c) cell size $0.2 \mathrm{~m}$. 
At free surface location relative dense mesh was created as this is a prerequisite to reduce wave height decay as wave propagated in the numerical wave tank.

An optimum mesh profile can efficiently play an important role in minimizing the reflection effect caused at the outer domain boundaries therefore a stretched cells structure was achieved adjacent to these far-end boundaries, a vertical slice of the mesh is shown in Fig. 9.

1.5.1.2. Boundary conditions. Boundary conditions applied to the CFD wave tank are explained in Table 4. To minimize wave reflection from the downstream (right hand side) end of the wave tank, the outflow boundary condition was applied together with stretched cells adjacent to this boundary.

1.5.1.3. Turbulence model. The RNG model was employed throughout all simulations unless stated otherwise. This model is based on the Renormalization Group (RNG) methods. In this approach the derivation of the turbulence quantities such as turbulent kinetic energy and the corresponding dissipation rate is accomplished using statistical methods.

The RNG model is based on the similar equations as the $k-\varepsilon$ model but the constants of these equations are found explicitly whereas in $k-\varepsilon$ model these coefficients are obtained empirically. RNG model is known to describe strong shear regions of the flow more accurately. The minimum value of the rate of the turbulent energy dissipation $\varepsilon_{T}$ is limited according to the following equation

$$
\varepsilon_{T, \min }=\mathrm{CNU} \sqrt{\frac{3}{2}} \frac{k_{T}^{3 / 2}}{\mathrm{TLEN}},
$$

where CNU is a parameter ( 0.09 by default), $k_{T}$ is the turbulent kinetic energy and TLEN is the turbulent length scale. A constant value for this length scale is chosen according to the rule of $7 \%$ of the dominating moving body's dimension. Further detail about underlying mathematical model and numerical scheme is available in [33].

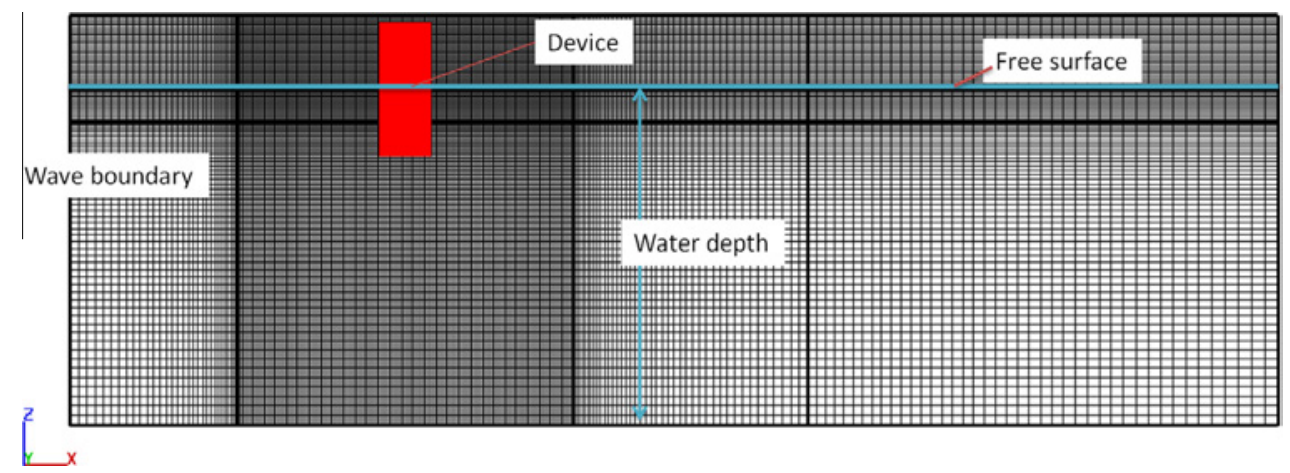

Fig. 9. Mesh profile of the computational domain of a CFD simulation for wave structure interaction.

Table 4

Boundary conditions explained.

\begin{tabular}{lll}
\hline Face Number & Face of NWT & Boundary condition \\
\hline 1 & Left $-X \min$ & Wall or wave boundary \\
2 & Right $-X \max$ & Outflow \\
3 & Front $-Y$ min & Symmetry \\
4 & Back $-Y$ max & Symmetry \\
5 & Top $-Z$ max & Fixed pressure \\
6 & Bottom $-Z$ min & Wall \\
\hline
\end{tabular}




\subsubsection{Convergence}

Solver convergence is controlled through automatic time step adjustment. This offered maximum possible time step size without compromising stability and convergence criteria. Pressure convergence is insured through defined number of iterations and in case convergence is not achieved within this limit then the solver is aborted (see [33]). In addition, mesh convergence check was performed. As discussed in [34], wave height attenuation can occur in CFD simulations therefore the optimum mesh structure employed in all simulations insured that the desired wave achieved at the device location is within a reasonable accuracy. Results for three different mesh structure are shown in Fig. 10a where device displacement in response to an incoming wave of amplitude $2 \mathrm{~m}$ and wave period $8 \mathrm{~s}$ is drawn, it can be seen that negligible differences are observed between each mesh however Mesh $_{\mathrm{F} 1}$ was used for the comparison. Mesh independence test for radiation force computation is shown in Fig. 10b where mesh refinement allows to reduce noise and spikes. Although further mesh refinement may be needed to fully resolve this issue. However, it is a peculiar effect occuring within limited time duration and it is observed that it does not affect the motion response and the overall aspect of the force is converged. Thus it is believed that it does not affect the estimates for $C_{\mathrm{d}}$.

CFD simulations for each case study (diffraction, radiation and WSI) comprise varying mesh structure depending on the corresponding requirements of each scenario. Cell size information of the mesh used for results of Fig. 10 is listed in Table 5. CPU time for a specific CFD simulation of WSI problem of $50 \mathrm{~s}$ wall clock time is given below:

Dimension of the computational domain: $190 \mathrm{~m}, 50 \mathrm{~m}, 60 \mathrm{~m}(x, y, z)$; total number of cells: 2,042,044; smallest cell size: 0.3; CPU time of a typical simulation: $8 \mathrm{~h} 8 \mathrm{~min}$; Computational resource: RAM: 6GB, processor: Intel(R) Xeon(R) CPU E5620 @2.40GHZ 2.39GHZ; system: Windows 7 Professional 64 bits.

\section{Radiation}

Radiation refers to the cases where the surface piercing profile of the wave energy converter is made to oscillate in otherwise calm fluid. The rocking structure then generates waves that appear

(a)
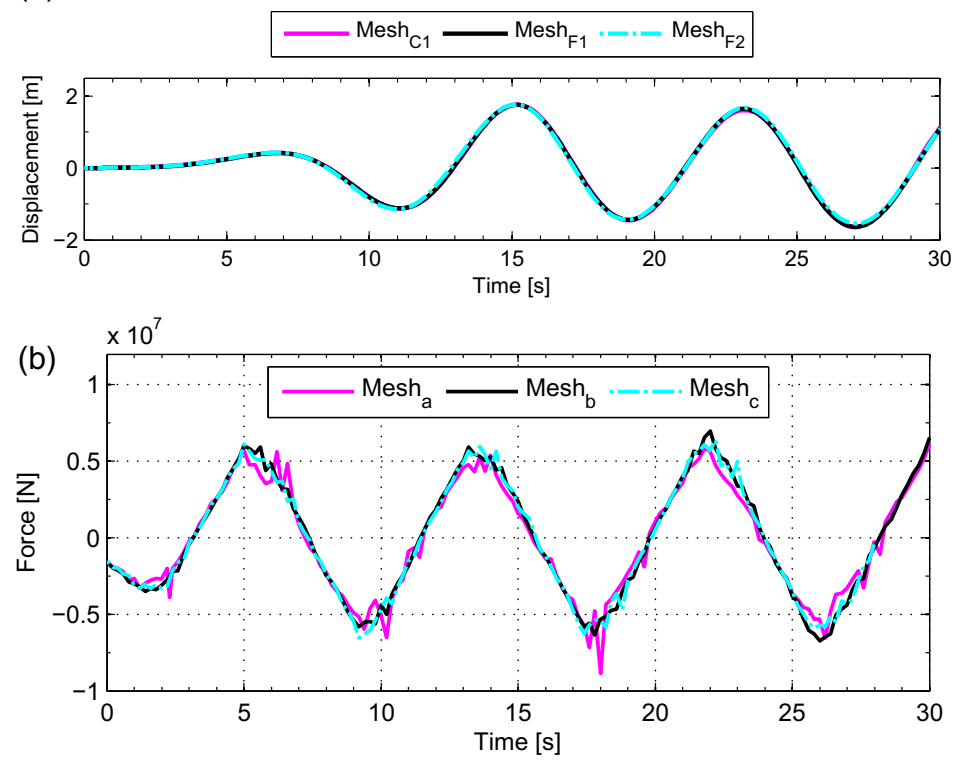

Fig. 10. Results of mesh convergence check for CFD simulation (a) displacement from coupled wave structure motion, (b) radiation force from radiation case study. 
Table 5

Mesh information for convergence test.

\begin{tabular}{llll}
\hline & Mesh & Min cell size $[\mathrm{m}] X$-axis & Total cells \\
\hline Coupled WSI & Mesh $_{\mathrm{C} 1}$ & 0.4 & 731,768 \\
& Mesh $_{\mathrm{F} 1}$ & 0.3 & $1,536,480$ \\
\multirow{3}{*}{ Radiation force } & Mesh $_{\mathrm{F} 2}$ & 0.2 & $2,705,039$ \\
& Mesh $_{\mathrm{a}}$ & 0.4 & $1,604,768$ \\
& Mesh $_{\mathrm{b}}$ & 0.3 & $2,630,700$ \\
& Mesh $_{\mathrm{c}}$ & 0.2 & $3,017,774$ \\
\hline
\end{tabular}

Table 6

CFD simulations for radiation tests of surging WEC.

\begin{tabular}{llll}
\hline Case study & Amplitude $[\mathrm{m}]$ & Period $[\mathrm{s}]$ & KC \\
\hline SB2 & 3 & 5 & 1.5 \\
SB3 & 5 & 8 & 4.0 \\
\hline
\end{tabular}

to radiate as the rigid body moves back and forth. Table 6 presents case studies considered for the CFD simulations of the surging WEC.

The amplitude and frequency shown in Table 6 refer to the amplitude and the frequency of the prescribed/imposed velocity. The last column of this Table show the corresponding KC number.

The in-line force from the above mentioned CFD simulations (Table 6) is compared against the results of the frequency domain analysis. The methodology of computing the radiation force from the frequency domain analysis is presented below. Following the computations from the BEM frequency domain in-house research code Aquaplus, the frequency dependent hydrodynamic coefficients $A, B$ and $F_{\mathrm{e}}$ are known, where; $A$ is added mass, $B$ is radiation damping, and $F_{\mathrm{e}}$ is the excitation force. It is worth noticing that the excitation force where a rigid structure is held still, in a wave climate, is also taken as being equivalent to the diffraction force.

Frequency domain radiation force coefficient $\left(F_{\mathrm{rad}}(\omega)\right)$ can be written as

$$
F_{\text {rad }}(\omega)=U_{\mathrm{m}}(i \omega A-B)
$$

Here $U_{\mathrm{m}}$ is the amplitude of the velocity of the moving structure. The corresponding time domain equivalent $F_{\text {rad }}(t)$ is:

$$
F_{\text {rad }}(t)=\mathfrak{R}\left(F_{\text {rad }}(\omega) e^{i \omega t}\right)
$$

Here $\mathfrak{R}$ refers to the real part of the complex value. Drag force is

Table 7

CFD simulations, wave diffraction cases.

\begin{tabular}{llll}
\hline Case study & Wave amplitude $[\mathrm{m}]$ & Wave period $[\mathrm{s}]$ & KC \\
\hline DB1 & 1 & 5 & 0.6 \\
DB2 & 2 & 8 & 1.2 \\
\hline
\end{tabular}

Table 8

CFD simulation case studies for regular waves.

\begin{tabular}{lll}
\hline Case study & Wave amplitude $[\mathrm{m}]$ & wave period $[\mathrm{s}]$ \\
\hline RWAB1 & 2 & 8 \\
RWAB2 & 3 & 10 \\
RWAB3 & 5 & 10 \\
\hline
\end{tabular}


Table 9

CFD simulation case studies for irregular waves.

\begin{tabular}{lll}
\hline Wave spectrum & Significant wave height $[\mathrm{m}]$ & Peak wave period $[\mathrm{s}]$ \\
\hline $\mathrm{Sf}_{1}$ & 2.5 & 7 \\
$\mathrm{Sf}_{2}$ & 3.5 & 9 \\
$\mathrm{Sf}_{3}$ & 5 & 12 \\
\hline
\end{tabular}

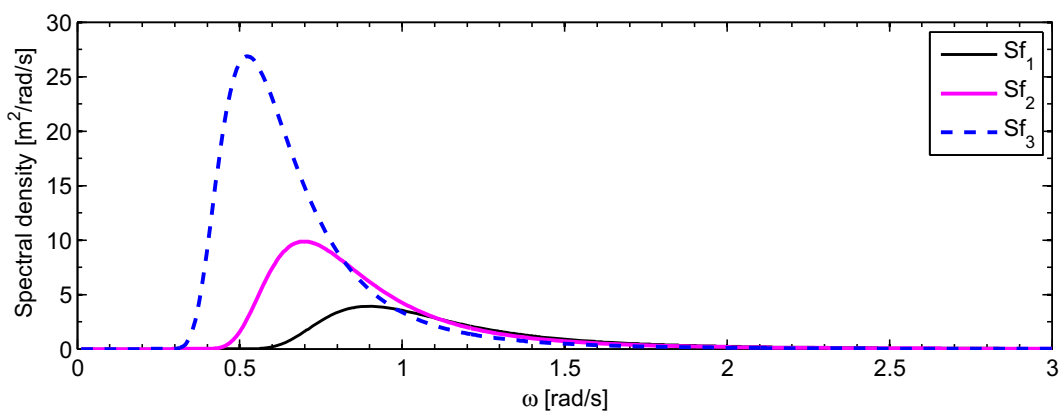

Fig. 11. Wave spectra for random wave scenarios.

(a)

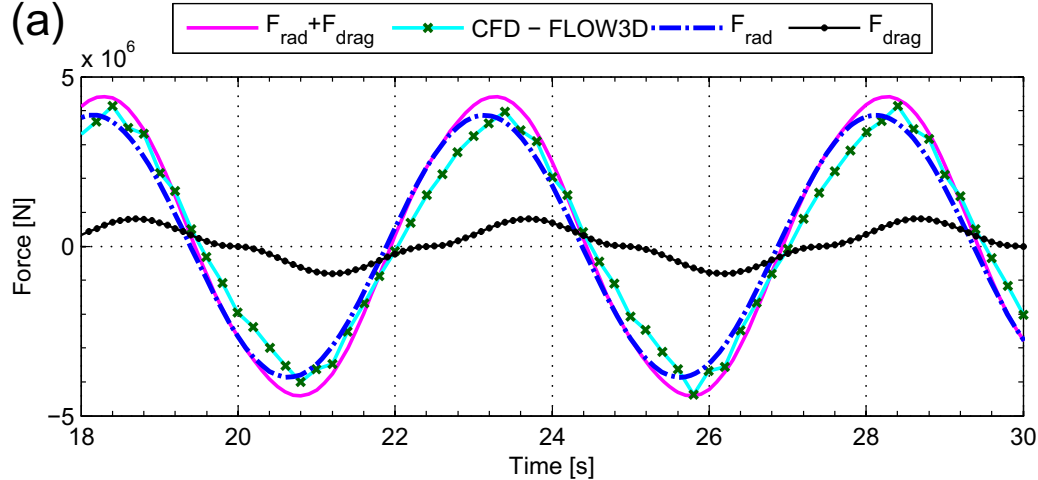

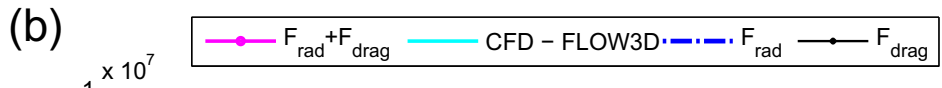

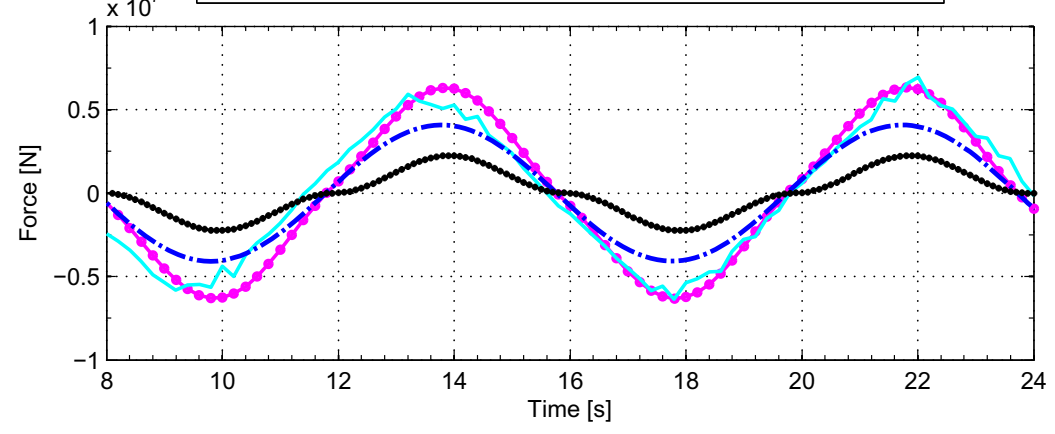

Fig. 12. Radiation force comparison for imposed surge motion; (a) case study SB2, (b) case study SB3. 


$$
F_{\text {drag }}(t)=-\frac{1}{2} \rho A C_{\mathrm{d}} \dot{X}|\dot{X}|
$$

The in line force obtained from the sum of Eq. (27) and Eq. (28), that is $F_{R D}(t)$ (Eq. (29)), is then compared to the total force obtained from the CFD results.

$$
F_{R D}(t)=F_{\text {rad }}(t)+F_{\text {drag }}(t)
$$

\section{Diffraction}

This section presents simulations of the cases where a wave energy converter is held still whilst the wave loadings are computed numerically. As the wave interacts with the still structure, a diffraction wave field emerges and the resulting in-line wave force is, in this case, termed as diffraction force and is denoted as $F_{\text {Diff }}$. The time history of the diffraction force is obtained from the excitation force coefficient evaluated using Aquaplus. Eq. (30) shows the calculation of the time series of the $F_{\text {Diff }}$.

$$
F_{\text {Diff }}(t)=A_{\text {wave }} \mathfrak{R}\left\{\left|F_{\mathrm{e}}(\omega)\right| e^{i \omega t}\right\}
$$

Here $A_{\text {wave }}$ is the amplitude of the incoming wave.

For this type of analysis the rigid device is non-moving. Performed CFD simulations are shown in Table 7. Results of these CFD simulations were then compared with the ones obtained through Eq. (30).

(a)
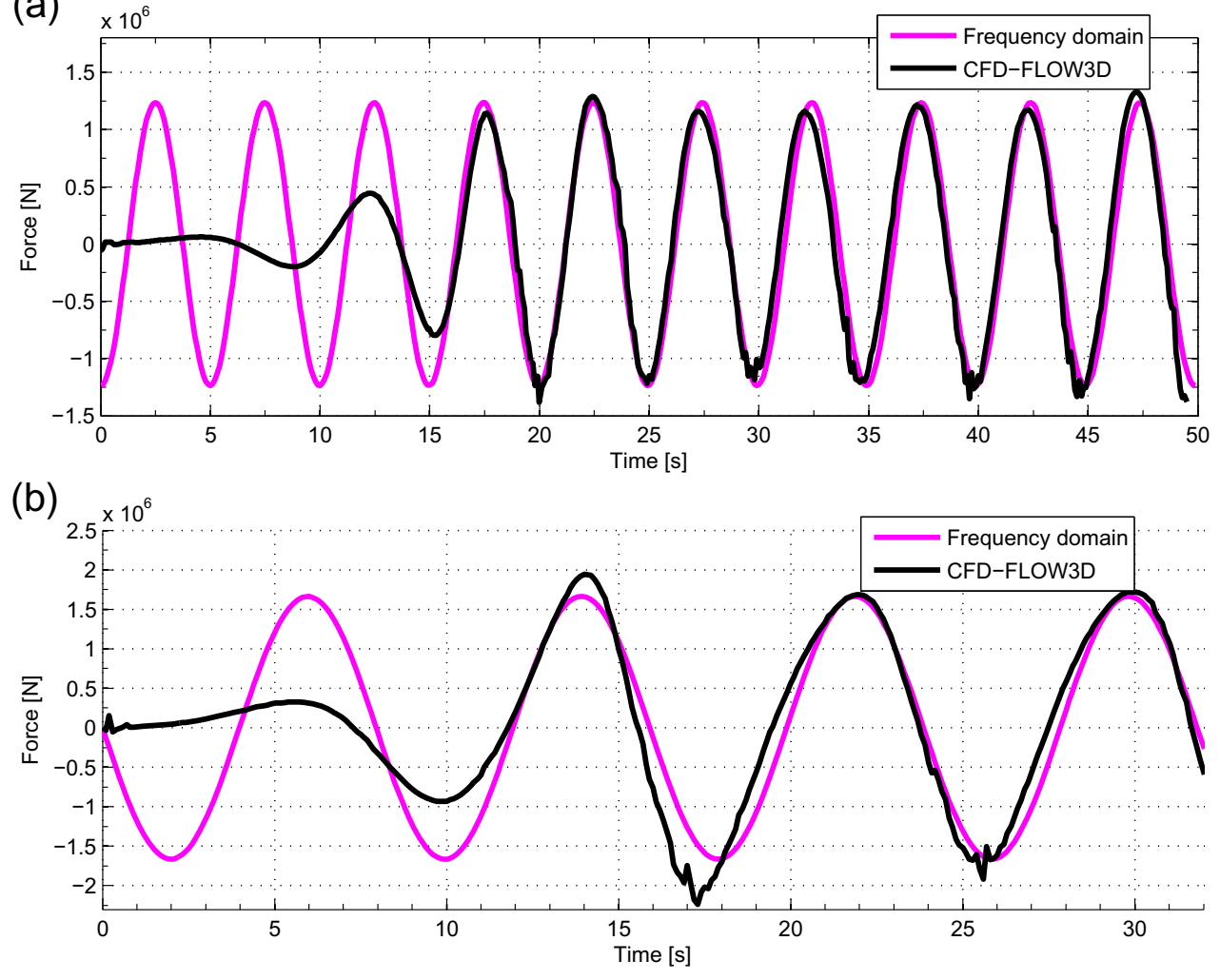

Fig. 13. In-line wave force - (a) case study DB1, (b) case study DB2. 


\section{Coupled wave structure interaction}

This section deals with the CFD case studies of the wave propagation and the resulting response of the wave energy device. Coupled wave structure interaction refers to a scenario where the wave energy converter is allowed to respond to a wave force of the incoming wave profile. Additional control

(a)

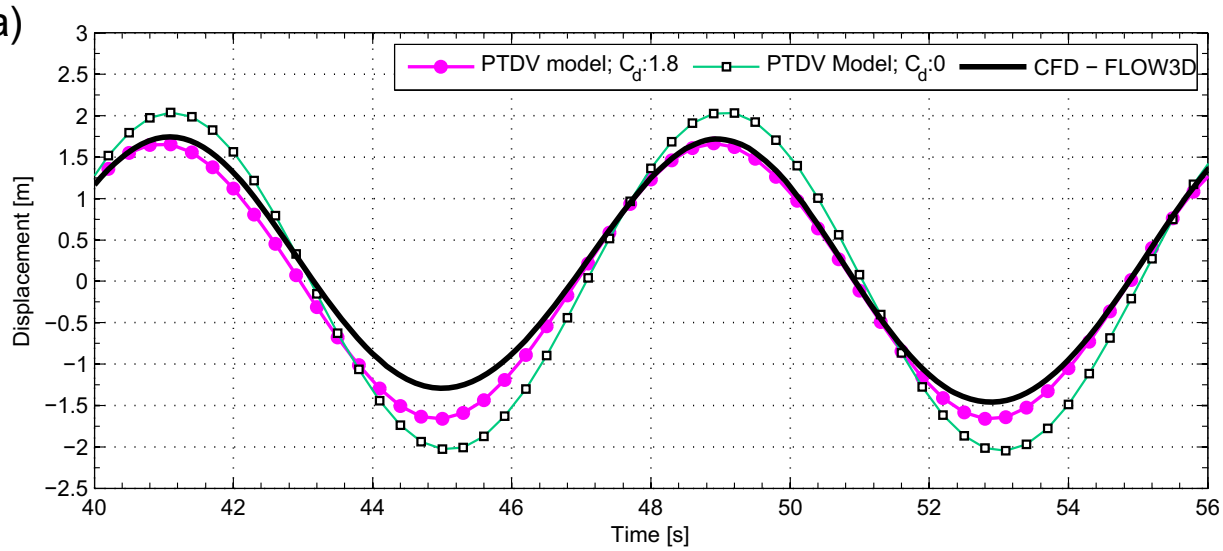

(b)

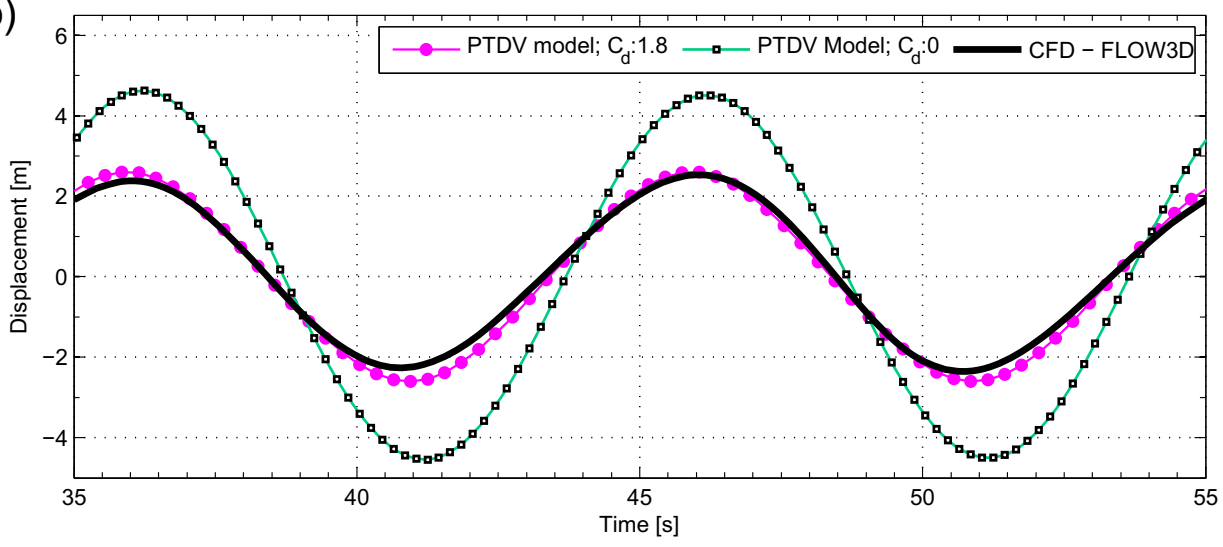

(c)

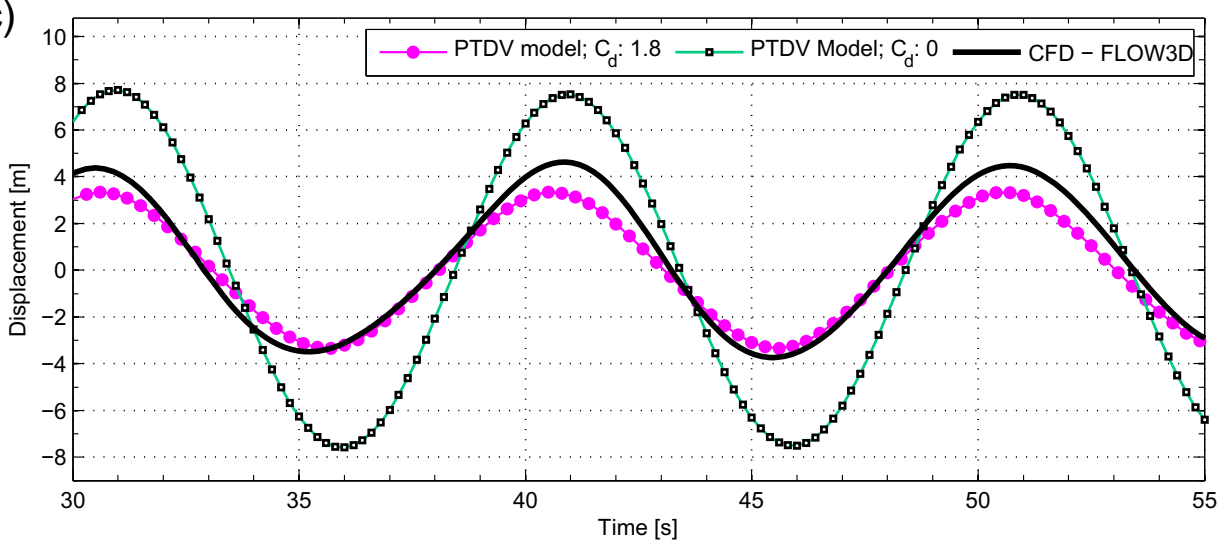

Fig. 14. Displacement of Surging WEC from CFD and PTDV mode, case study; (a) RWAB1, (b) RWAB2, and (c) RWAB3. 
forces like power-take-off damping as a function of the velocity and restoring force which is a function of the displacement were added into the CFD model. This was achieved by customization of the relevant Fortran source code of the FLOW3D. The code was then recompiled and the customized version was used for rest of the simulations presented here on wards. This additional power take off and restoring force can be written in the form of the following Eq. (31):

$$
f_{x}=-B_{\mathrm{pto}} \dot{X}-K_{11} X
$$

Here $B_{\text {pto }}$ is the power take off damping coefficient, and $K_{11}$ is the restoring (stiffness) coefficient. $X, \dot{X}$ being the position and velocity of the body mass center, respectively.

For the CFD analysis a numerical wave tank was established and the wave energy device was tested in regular (sinusoidal) monochromatic and irregular (random) incident waves. Resulting instantaneous displacement of the WECs was then compared with the outcome of the PTDV model. In doing so a comprehensive inspection of the methodology employed in PTDV is conducted. The nominal value of the drag coefficient is used for this comparison.

CFD simulation setup in these cases comprised a wave boundary specified at the left (minimum $X$ axis) end of the wave tank. Whereas the far end boundary (maximum $X$ axis) was set to the available option of the outflow boundary which allowed the circulating fluid to re-enter the fluid domain hence minimizing the wave reflection effect. Moreover as in other cases of the CFD simulations the mesh at this far end boundary was set to a bigger cell value, this enabled the achievement of a gradually stretched cell structure towards this outflow boundary. Whereas the mesh cells at the wave boundary was kept to a smaller cell value so that to aid the wave propagation in order to diminish the wave height decay. This issue of wave propagation regarding commercial CFD packages including FLOW3D

(a)

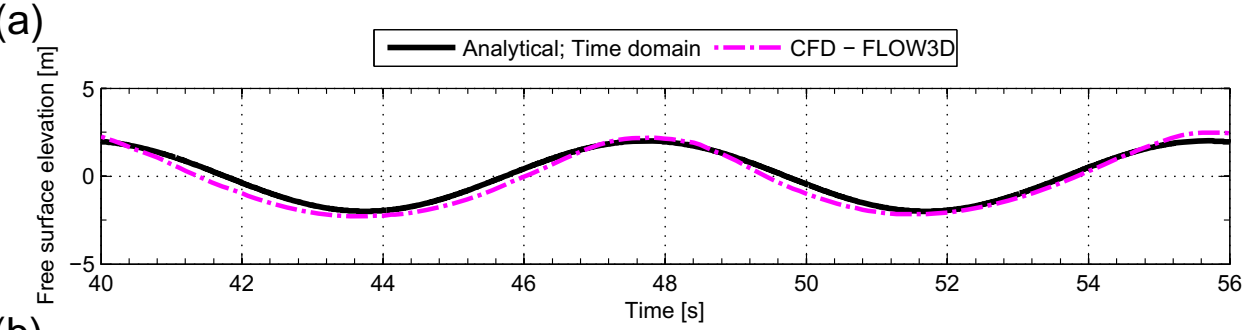

(b)

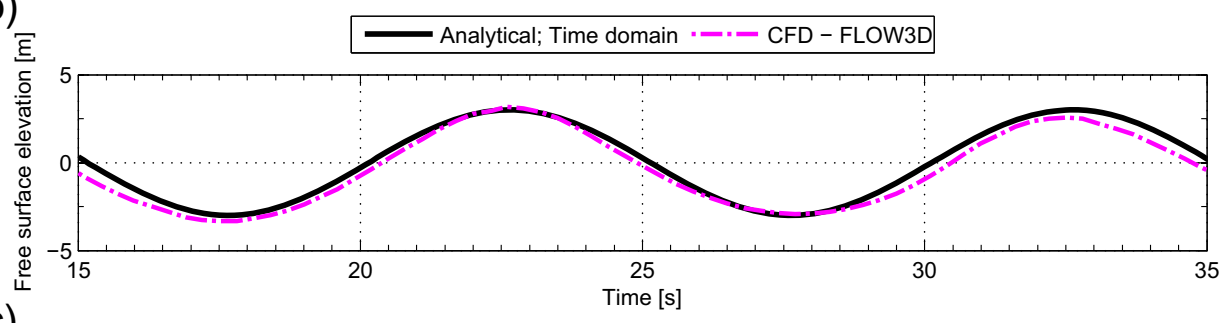

(C)

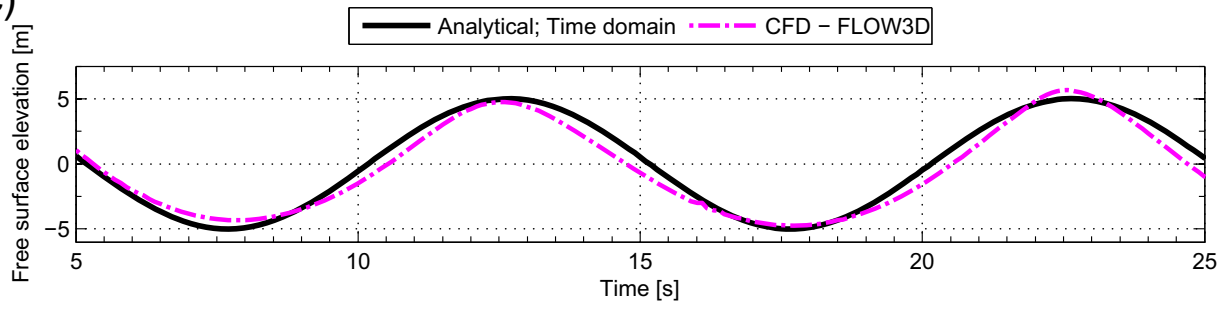

Fig. 15. Free surface elevation; CFD results against analytical results of time domain model, case study; (a) RWAB1, (b) RWAB2, and (c) RWAB3. 
has also been pointed by [34]. In order to address this issue, the wave energy converter was placed slightly closer to this wave boundary rather than in the middle of the tank.

\subsection{Regular waves}

Case studies of CFD simulations of the wave energy converters in regular (sinusoidal) incoming wave climate are shown in Table 8. Through these case studies, motion of the surging wave energy converter against linear waves is studied via CFD analysis. The CFD computations are then examined against the one obtained through state of the art methodology: PTDV model.

\subsection{Irregular (or random) waves}

This section describes CFD and PTDV model results for a set of irregular monochromatic waves. Like regular wave case studies the PTO damping force and additional stiffness, as explained in Section 4 , are included into the CFD model. Irregular wave is defined according to the Bretschneider spectrum. Table 9 shows the input wave parameters for each numerical test case. Irregular wave spectrum for each numerical case study is shown in Fig. 11. This wave spectrum and the angular frequencies were used as input parameters to the FLOW3D and it was observed that random wave being generated by FLOW3D is based on the amplitude which are given by

$$
A(\omega)=\sqrt{2\left(\mathrm{Sf}_{k}\right) \Delta \omega}
$$

(a)

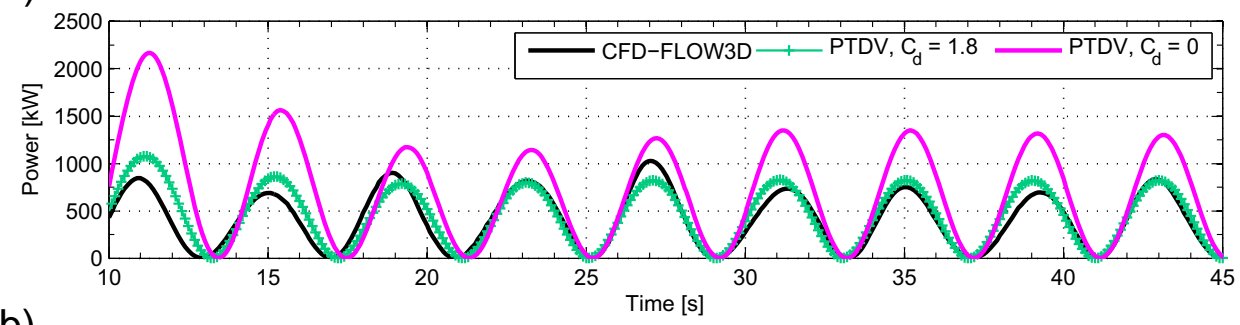

(b)

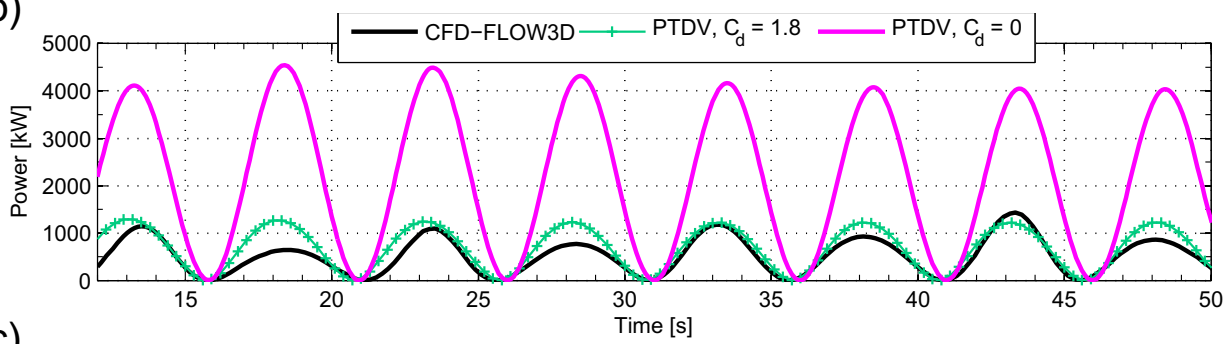

(c)

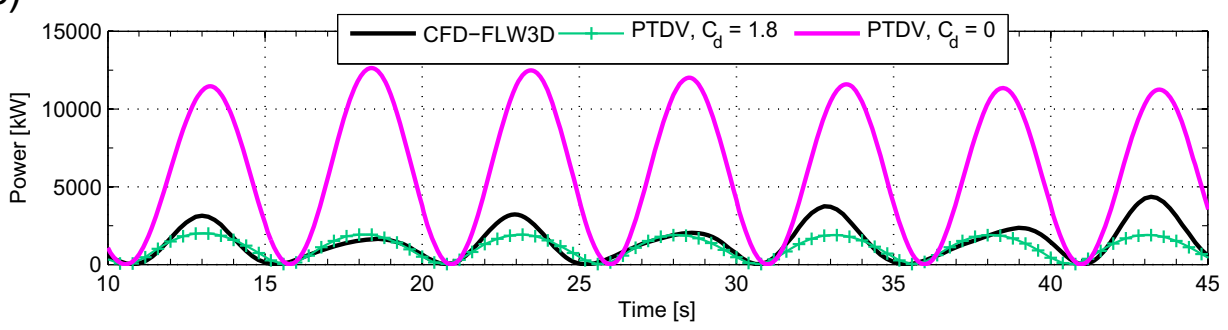

Fig. 16. Instantaneous power for case study: (a) RWAB1, (b) RWAB2, (c) RWAB3. 
Here $\omega$ is the angular velocity in $\mathrm{rad} / \mathrm{s}, \mathrm{Sf}_{k}$ is the spectrum corresponding to the individual case study for $k=1,2,3$ and $\Delta \omega$ is the angular frequency step for the frequency points.
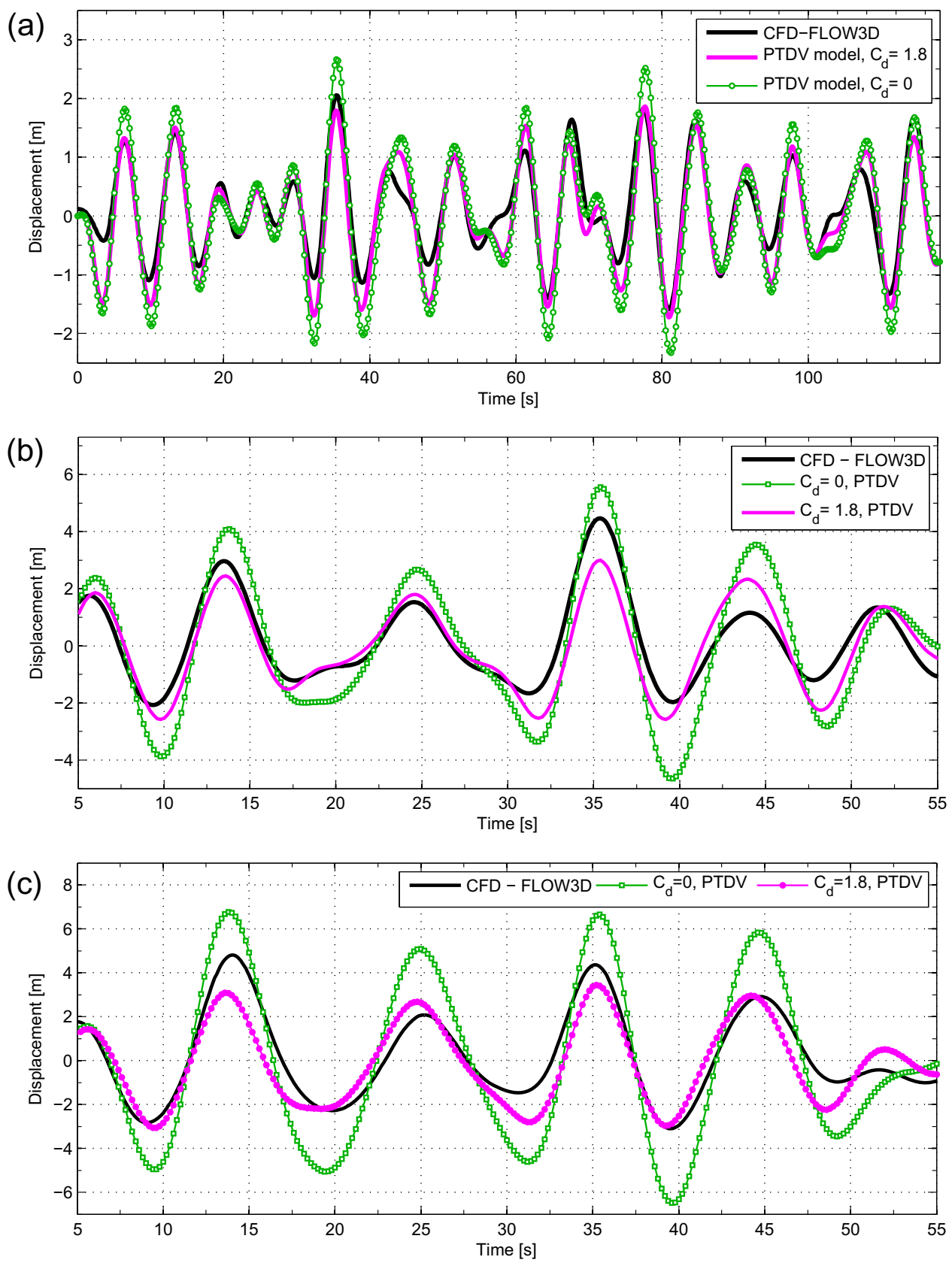

Fig. 17. Displacement corresponding to wave spectrum; (a) $\mathrm{Sf}_{1}$, (b) $\mathrm{Sf}_{2}$, (c) $\mathrm{Sf}_{3}$. 


\section{Results}

\subsection{Radiation}

Results from the sum of the radiation and drag force are compared against the CFD data for the surging WEC. The surging WEC described earlier was made to oscillate at various sets of sinusoidal velocities illustrated in Table 6. CFD simulations were set-up so that the free surface response can be taken into account. The radiated waves were then generated and the resulting radiation force is investigated alongside the radiation force obtained via frequency domain model.

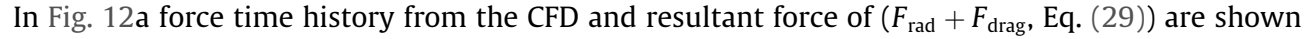
for test SB2. Although for this specific case of small amplitude linear monochromatic wave, the drag force is relatively small as compared to the radiation force and therefore the corresponding sum of the drag force and the radiation force offers only a slight increment and is able to capture the peak of force curve as observed in the CFD model.

Radiation force comparison for SB3 is presented in Fig. 12b where It can be seen that the addition of the drag term offers an improved force prediction as the upper limit of the CFD force amplitude is recovered by the additional drag term. It seems that for relatively large KC value the surrounding fluid becomes more turbulent and coupling of the low pressure vortex formation and the possible reflection from the outer boundaries appear to be as a major reason for the high frequency noise on the in-line force curve.

(a)

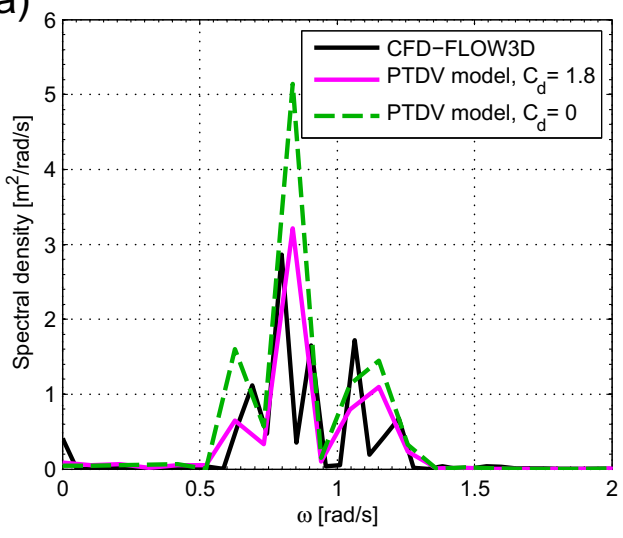

(b)

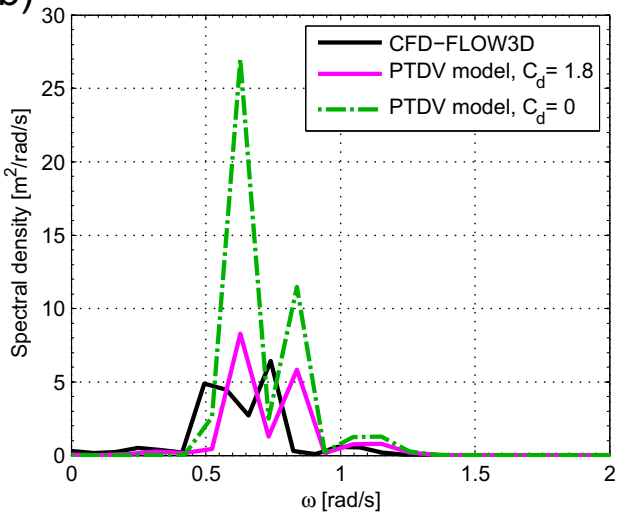

(c)

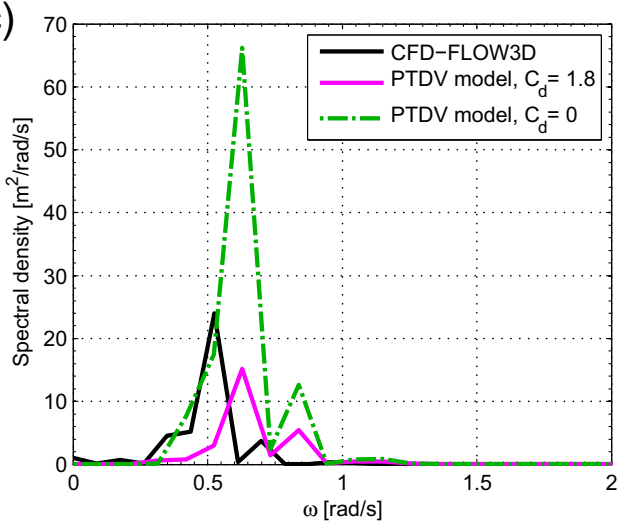

Fig. 18. Spectral density form displacement time series; (a) $\mathrm{Sf}_{1}$, (b) $\mathrm{Sf}_{2}$, (c) $\mathrm{Sf}_{3}$. 


\subsection{Diffraction}

Results of case studies DB1 and DB2 are shown in the form of the wave force in Fig. 13(a and b, respectively). In Fig. 13 the diffraction force which comes through the Eq. (30) is labeled as Frequency domain whereas the CFD-FLOW3D is the total in-line force computed via CFD simulations of FLOW3D. A reasonably good comparison of the two force curves raises confidence in the frequency domain approach that for such a small amplitude motion the linear potential model provides competitive results when compared to the RANSE solver.

Fig. 13b shows diffraction force comparison for the case study of relatively higher wave amplitude. Here the incoming wave corresponds to the case study DB2. A reasonably good comparison for this case of relatively higher KC number flow is evident.

\subsection{Coupled wave structure interaction}

\subsubsection{Regular wave}

Fig. 14 show displacement of the surging WEC for three different wave conditions (Table 8), respectively. Fig. 14a shows displacement of the surging wave energy converter for a regular wave of amplitude $2 \mathrm{~m}$ and time period $8 \mathrm{~s}$. PTDV model results with and without drag force; (that is $C_{\mathrm{d}}: 1.8$ and 0 ); are shown along corresponding displacement curves from the CFD model. It is quite prominent that in

(a)

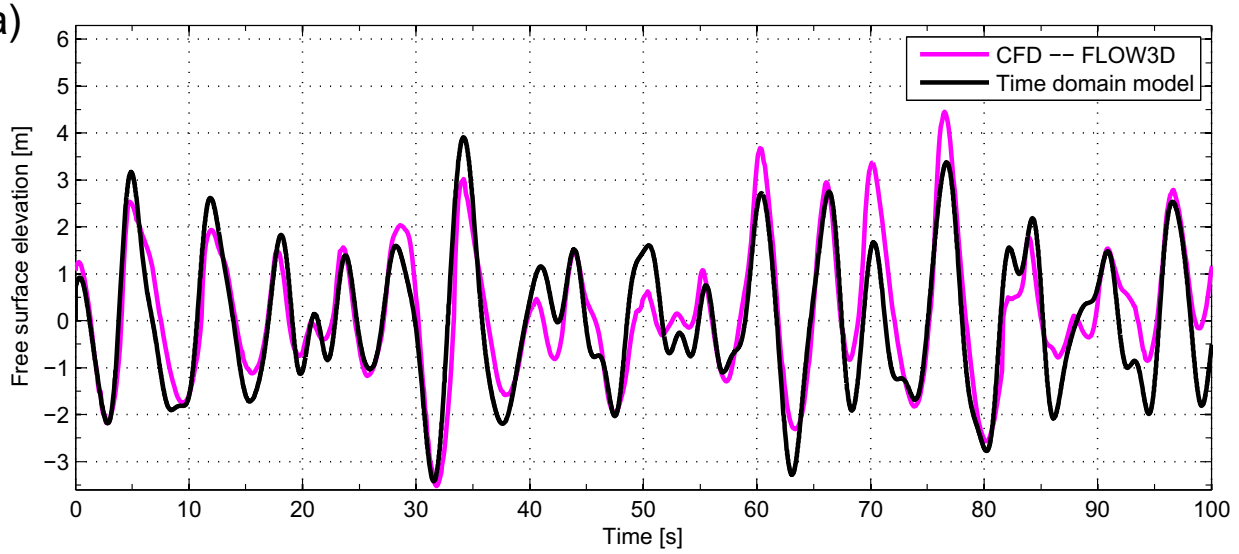

(b)

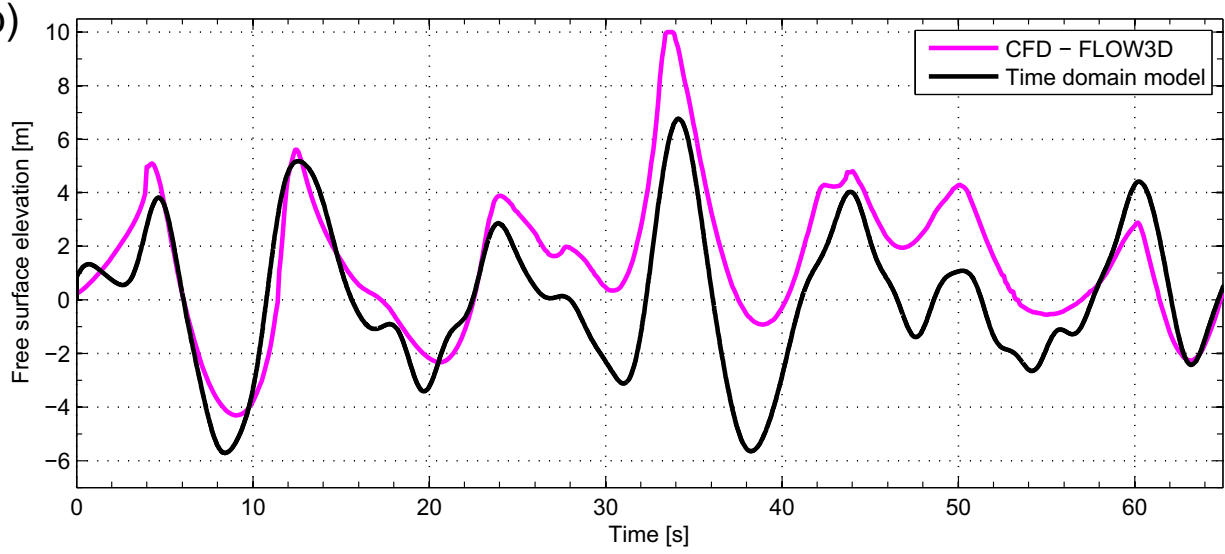

Fig. 19. Free surface elevation; (a) case study $\mathrm{Sf}_{1}$, (b) case study $\mathrm{Sf}_{3}$. 
the absence of the drag force (for $C_{d}=0$ ) the PTDV model is over-estimating the response of the device and hence, it was leading to a higher estimate of the power output.

Fig. 14b presents displacement of the surging device for case study RWAB2. It is shown that the drag value of 1.8 insures good agreement when compared to the CFD lead results. Similar conclusions are observed for case study RWAB3 as seen in Fig. 14c where displacement of the surging WEC in response to the wave forces of a linear wave of amplitude $5 \mathrm{~m}$ and wave period $10 \mathrm{~s}$ is shown. $C_{\mathrm{d}}$ value of 1.8 is shown to produce better agreement with the CFD output.

Wave profiles from both numerical models are shown in Fig. 15. Here the corresponding wave elevation from all the three simulations from CFD wave tank and the analytical wave elevation from the time domain model is presented. It can be seen that the wave elevation from CFD model is asymmetrical which is a typical feature of the nonlinearity and therefore show deviations from the linear model.

(a)

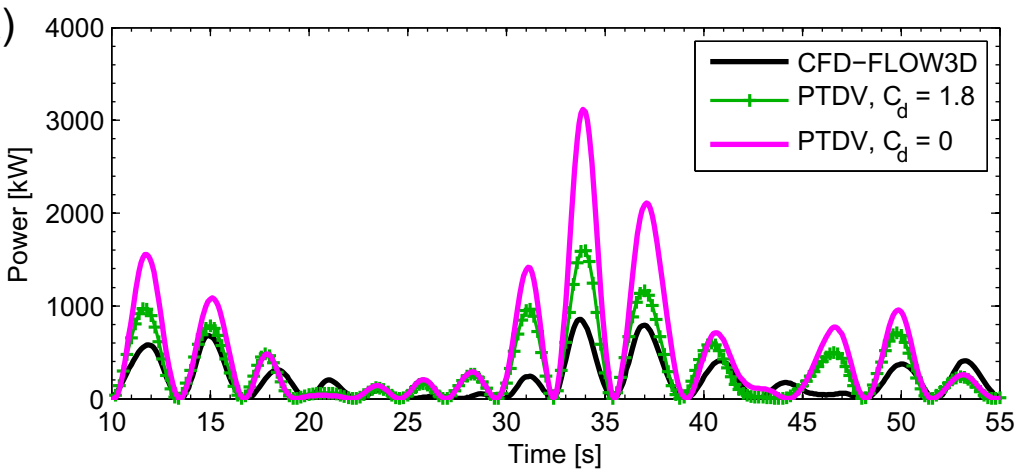

(b)

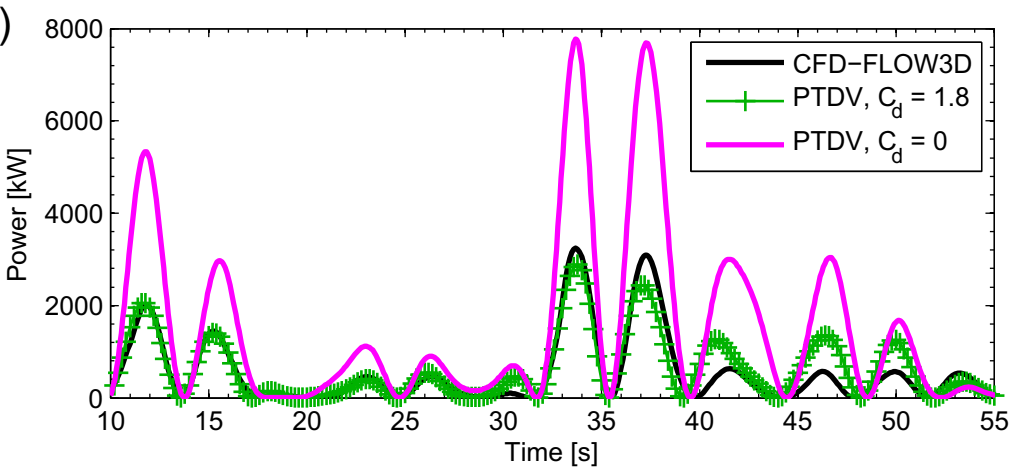

(c)

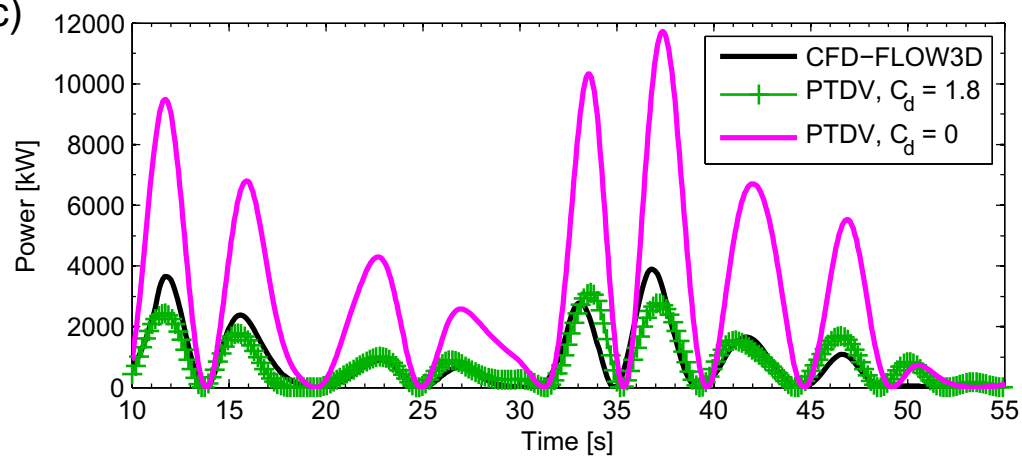

Fig. 20. Instantaneous power for random wave: (a) $\mathrm{Sf}_{1}$; (b) $\mathrm{Sf}_{2}$; (c) $\mathrm{Sf}_{3}$. 
Fig. 16(a-c) are the corresponding instantaneous power curves for the three test cases; it can be seen that the PTDV model (with a suitable $C_{d}$ value) reduces the over-estimation of the power production to a greater extent. The mean power value for each above mentioned case studies (RWAB1, RWAB2 and RWAB3) are shown with the help of a bar-plot in Fig. 21 which concludes that the PTDV model carries a capability of predicting the power output competently when compared to the CFD simulation output.

\subsubsection{Irregular or random waves}

Fig. 17 shows time history of the instantaneous position of the surging wave energy converter for the three cases $\left(\mathrm{Sf}_{1}, \mathrm{Sf}_{2}\right.$ and $\left.\mathrm{Sf}_{3}\right)$. It can be observed that the drag damping force when included into time domain model offers a considerable improvement.

For the first case of wave spectrum $\mathrm{Sf}_{1}$, device displacement from all models is shown in Fig. 17a, considering the complexity in generating random wave in CFD simulations, a good agreement with modified time domain model is reported.

Fig. 17b shows the displacement of the surging WEC for the second case study where a random wave field from spectrum $\mathrm{Sf}_{2}$ was generated. The time domain results for two values of the drag coefficient i.e., $C_{\mathrm{d}}=0, C_{\mathrm{d}}=1.8$ are plotted alongside the CFD findings. Here $C_{\mathrm{d}}=1.8$ corresponds to a nominal value of the drag coefficient calculated via CFD [13]. Results shown in this Fig. 17b highlight a number of important aspects of the CFD and the PTDV model. It is noted that, firstly, the PTDV model over-estimates the motions of the WEC in the absence of the additional drag force. Secondly, when the drag force corresponding to the nominal value of the $C_{\mathrm{d}}$ is included into the PTDV model the comparison with the CFD is reasonably quite good for the first $30 \mathrm{~s}$ of wave structure interaction. This is because the wave amplitude and hence the motion amplitude of the device were relatively small. Thirdly, when the wave with much greater height approaches, at $t=32 \mathrm{~s}$ to $40 \mathrm{~s}$, the resulting peak of the displacement amplitude undergoes a sudden jump. In this case the displacement peak obtained through PTDV model with $C_{\mathrm{d}}$, although an improvement, but is still about $40 \%$ under-estimation as compared to the CFD data. Resulting claim of the PTDV model can also be demonstrated by the Fig. 20 where time history of the power output is shown.

Results of the third wave spectrum $\mathrm{Sf}_{3}$ are shown through Fig. 17c. Looking at the device displacement with and without the drag term at time instants of $20 \mathrm{~s}$ and $30 \mathrm{~s}$, it can be noted that the addition of the viscous drag not only reduces the extent of the over-estimation of the device motion but also introduces nonlinearity in the resultant motion like the one shown by CFD simulations.

Spectral density of the device displacement is also shown for the three irregular wave cases; see Fig. 18a, b and c, respectively. Certain discrepancy in the spectral density may also be due to the number of data points available from each simulation.

The surface elevation of wave propagation for the two simulations $\left(\mathrm{Sf}_{1}\right.$ and $\left.\mathrm{Sf}_{3}\right)$ of irregular wave are shown in Fig. 19a and b. As the significant wave height of $5 \mathrm{~m}$ with a peak wave period of $12 \mathrm{~s}$ pro-

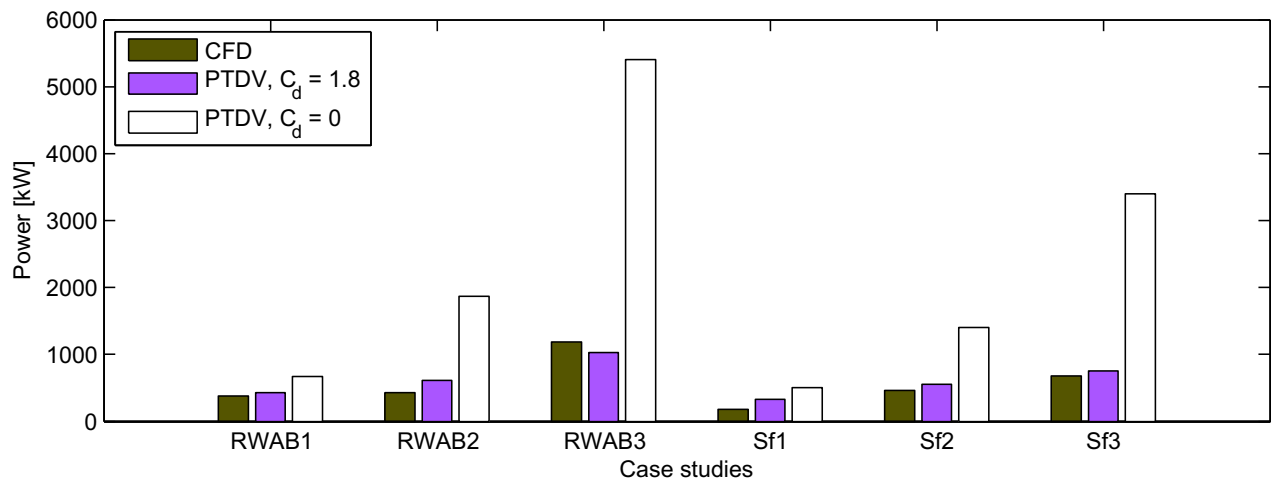

Fig. 21. Mean power for case studies of regular and random wave spectra. 
duces relatively energetic random wave which is beyond the scope of the linear potential approach. Therefore the comparison of free surface elevation from CFD verses analytical linear model are tend to show certain discrepancies. However it can be seen from Fig. 20c that the instantaneous power output show similar trend as in previous case studies.

To further illustrate these outcomes and to aid the conclusive remarks the instantaneous power profile from the both numerical tools - the CFD and the PTDV model - is presented in Fig. 20. And the correspond mean power output all cases of coupled motion is shown given in Fig. 21.

Contours of turbulent energy from CFD simulations of wave spectrum $\mathrm{Sf}_{2}$ are presented in Fig. 22 where wave structure interaction for a sequence of four time instants are shown, it can be seen that some extent of wave breaking is also considered in CFD. Furthermore it is worth mentioning that the CFD model computes fluid forces on the instantaneous position of the moving object whereas in the presented PTDV model fluid loading calculation is made with reference to the mean position of the WEC. This can be another major reason of the small differences observed.

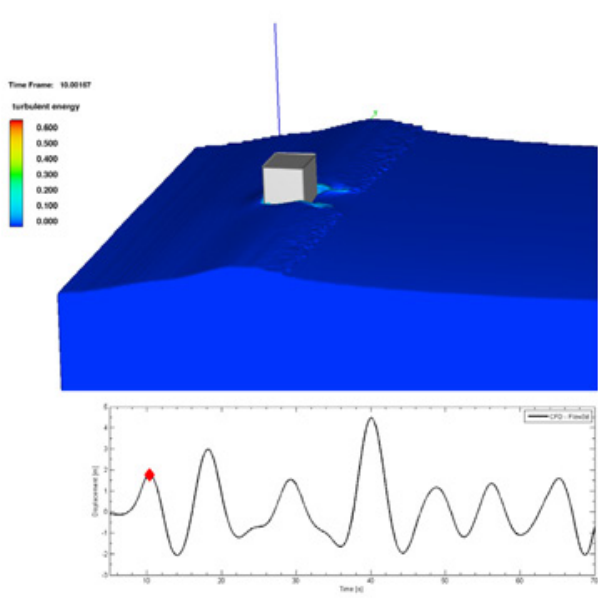

(a) t:10s
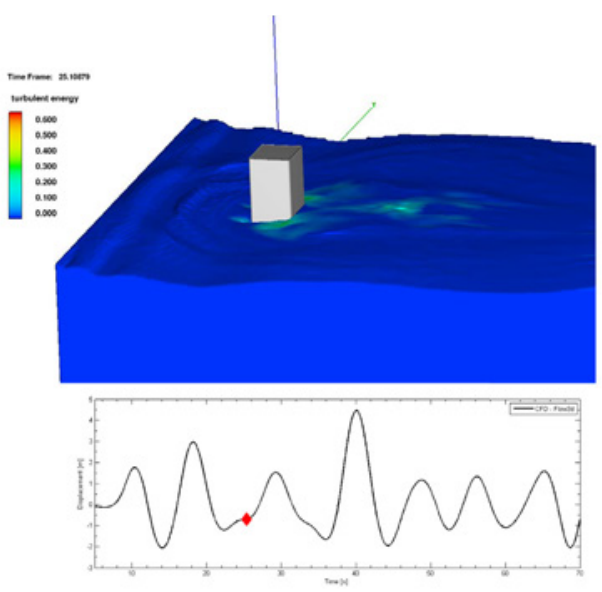

(c) $t: 25 s$
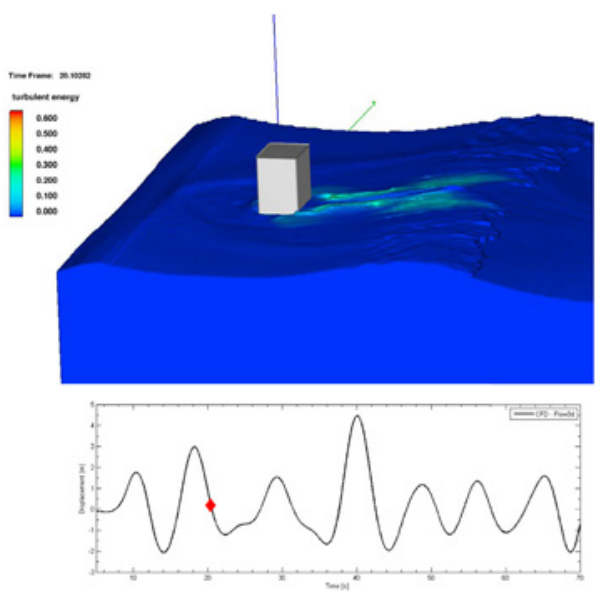

(b) t:20s
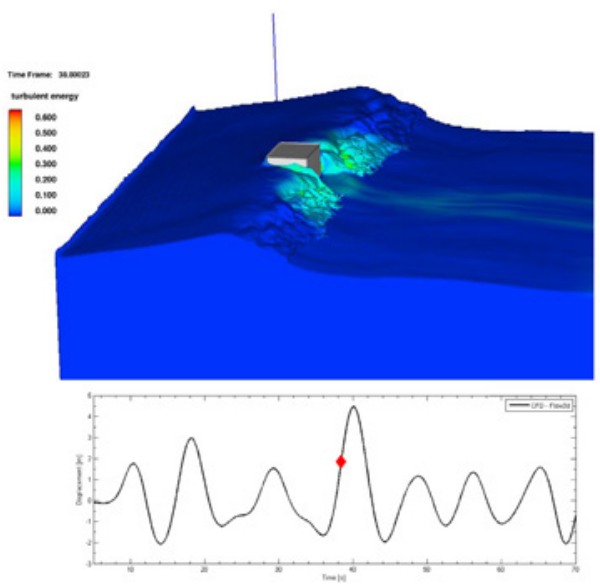

(d) t:38s

Fig. 22. CFD contour profiles showing turbulent energy corresponding to case study $\mathrm{Sf}_{2}$. Below each contour profile the diamond shaped mark on the displacement curve of WEC shows the corresponding instantaneous position of the WEC. 


\section{Conclusions}

Using wave to wire model it is shown that the APP is prone to the magnitude of the drag damping. Once this drag damping force is quantified for a given device a further tuning of the power take off damping may improve the power output (see Fig. 7).

The radiation force from the frequency domain model is found to be an under-estimation as compared to the CFD results. The inclusion of the drag term (Eq. (28)) into the radiation force $F_{\text {rad }}$ leads to a considerable improvement.

For wave diffraction scenario, it is observed that the CFD results are in accordance with the potential theory based frequency domain model for a small amplitude incident wave field. This serves a validation case study for the CFD model. However as the amplitude of the incident wave increases the difference between the two methodologies appear more widened.

For regular waves, a good agreement of CFD and PTDV model is observed. In case of irregular wave scenario a number of key points are noticed: for relatively smaller amplitude waves (amplitude $\leqslant 3 \mathrm{~m}$ ) the resulting displacement is within a range of $\leqslant 1.5 \mathrm{~m}$ and the numerical results obtained through the addition of the drag force into the state of the art wave to wire model show good agreement. It is also shown that without the addition of the drag term the displacement of the buoy is being overestimated which in-turn gives an amplified APP value of the device. For those parts of the irregular waves where the wave amplitude is relatively larger (i.e., in the range of wave amplitude $\geqslant 3 \mathrm{~m}$ ) the difference between the time domain model and the CFD is quite significant.

A methodology for identification and incorporation of the drag damping force in potential flow based models is presented and is shown to be in reasonable agreement with CFD simulations. Although comparison of PTDV and CFD results is not perfect at every instant; inclusion of quadratic drag damping of the Morison force is shown to control the APP over-estimation to a considerable extent, concluding that the methodology can be applied in practice for improving power output predictions of the wave to wire model of WECs.

\section{Acknowledgment}

This work was accomplished within the Marie Curie Initial Training Network wavetrain2, financed by the FP7 of the European Commission (contract-N MCITN-215414). This work was also supported by the project ANR11-MONU-018-01 MONACOREV of the French Agence Nationale de la Recherche (ANR).

\section{References}

[1] D. Ross, Power From Sea Waves, Oxford University Press, United Kingdom, 1996.

[2] A. Clément, P. McCullen, A. Falcão, A. Fiorentino, F. Gardner, K. Hammarlund, G. Lemonis, T. Lewis, K. Nielsen, S. Petroncini, et al, Wave energy in Europe: current status and perspectives, Renew. Sustain. Energy Rev. 6 (5) (2002) 405-431.

[3] S. Salter, J. Taylor, N. Caldwell, Power conversion mechanisms for wave energy, Proc. Inst. Mech. Eng., Part M: J. Eng. Maritime Environ. 216 (1) (2002) 1-27.

[4] J. Falnes, A review of wave-energy extraction, Mar. Struct. 20 (4) (2007) 185-201.

[5] B. Drew, A. Plummer, M. Sahinkaya, A review of wave energy converter technology, Proc. Inst. Mech. Eng., Part A J. Power Energy 223 (8) (2009) 887-902.

[6] A. Falcão, Wave energy utilization: A review of the technologies, Renew. Sustain. Energy Rev. 14 (3) (2010) 899-918.

[7] J. Hals, R. Taghipour, T. Moan, Dynamics of a force-compensated two-body wave energy converter in heave with hydraulic power take-off subject to phase control, in: Proceedings of the 7th European Wave and Tidal Energy Conference, Porto, Portugal, 2007.

[8] M. Folley, T. Whittaker, J. Van't Hoff, The design of small seabed-mounted bottom-hinged wave energy converters, in: Proceedings of the 7th European Wave and Tidal Energy Conference, Porto, Portugal, 2007.

[9] A. Babarit, J. Hals, M. Muliawan, A. Kurniawan, T. Moan, J. Krokstad, Numerical benchmarking study of a selection of wave energy converters, Renewable Energy 41 (2012).

[10] X. Chen, W. Duan, Multi-domain boundary element method with dissipation, J. Mar. Sci. Appl. 11 (1) (2012) 18-23.

[11] J. Journee, W. Massie, Offshore Hydromechanics, Delft University of Technology, 2001.

[12] M.A. Bhinder, A. Babarit, L. Gentaz, P. Ferrant, Assessment of viscous damping via 3 D-CFD modelling of a floating wave energy device, in: 9th European Wave and Tidal Energy Conference EWTEC, Southampton, UK, 2011.

[13] M.A. Bhinder, A. Babarit, L. Gentaz, P. Ferrant, Effect of viscous forces on the performance of a surging wave energy converter, in: Proceedings of the Twenty-second International Offshore and Polar Engineering Conference, Rhodes, Greece, 2012. 
[14] G.G. Stokes, On the Effect of the Internal Friction of Fluids on the Motion of Pendulums, vol. 9, Pitt Press, 1851.

[15] T. Sarpkaya, A critical assessment of Morison's equation, in: International Symposium on Hydrodynamics in Ocean Engineering. Preprints, vol. 1, 1981, pp. 447-468.

[16] T. Sarpkaya, Vortex shedding and resistance in harmonic flow about smooth and rough circular cylinders at high reynolds numbers, Tech. rep., DTIC Document, 1976.

[17] J. Morison, M. O'Brien, J. Johnson, S. Schaaf, The force exerted by surface waves on piles, Petrol. Trans. 189 (1950) 149-154.

[18] B. Sumer, J. Fredsøe, Hydrodynamics Around Cylindrical Structures, vol. 26, World Scientific Pub Co Inc., 2006.

[19] G. Keulegan, L. Carpenter, Forces on Cylinders and Plates in an Oscillating Fluid, US Department of Commerce, National Bureau of Standards, 1958.

[20] G. Cook, The lighthill correction to the Morison equation (Ph.D. thesis), Johns Hopkins University, 1987.

[21] T. Sarpkaya, Morison's equation and the wave forces on offshore structures., Tech. rep., DTIC Document, 1981.

[22] J. Lighthill, Waves and hydrodynamic loading, in: Proceedings of the Second International Conference on the Behaviour of Off-Shore Structures, held at Imperial College, London, England, 1979.

[23] Z. Yuan, Z. Huang, An experimental study of inertia and drag coefficients for a truncated circular cylinder in regular waves, J. Hydrodyn., Ser. B 22 (5) (2010) 318-323.

[24] P. Bearman, M. Downie, J. Graham, E. Obasaju, Forces on cylinders in viscous oscillatory flow at low Keulegan-Carpenter numbers, J. Fluid Mech. 154 (1) (1985) 337-356.

[25] C. Chen, F. Fang, Y. Li, L. Huang, C. Chung, Fluid forces on a square cylinder in oscillating flows with non-zero-mean velocities, Int. J. Numer. Methods Fluids 60 (1) (2009) 79-93.

[26] W. Zheng, C. Dalton, Numerical prediction of force on rectangular cylinders in oscillating viscous flow, J. Fluids Struct. 13 (2) (1999) 225-249.

[27] P. Bearman, J. Graham, E. Obasaju, G. Drossopoulos, The influence of corner radius on the forces experienced by cylindrical bluff bodies in oscillatory flow, Appl. Ocean Res. 6 (2) (1984) 83-89.

[28] S. Atluri, A. Magee, K. Lambrakos, CFD as a design tool for hydrodynamic loading on offshore structures, in: Proceedings of the 28th International Conference on Offshore Mechanics and Arctic Engineering, Honolulu, Hawaii, 2009.

[29] W. Cummins, The impulse response function and ship motions, Tech. rep., DTIC Document, 1962.

[30] A. Babarit, G. Duclos, A. Clément, Comparison of latching control strategies for a heaving wave energy device in random sea, Appl. Ocean Res. 26 (5) (2004) 227-238.

[31] G. Delhommeau, Seakeeping codes Aquadyn and Aquaplus, in: 19th WEGEMT School Numerical Simulation of Hydrodynamics: Ships and Offshore Structures, 1993.

[32] C. Hirt, B. Nichols, Volume of fluid (vof) method for the dynamics of free boundaries, J. Comput. Phys. 39 (1) (1981) $201-$ 225.

[33] FLOW3D Manual, V10.0.2.05, Flow Science Inc. Santa Fe, NM, USA, 2012.

[34] A. Maguire, Hydrodynamics, control and numerical modelling of absorbing wavemakers (Ph.D. thesis), The University of Edinburgh, 2011. 